\title{
Wieviel Florida steckt in Niedersachsen? Zur empirischen Evidenz der „Kreativen Klasse“ in einem deutschen Flächenland
}

\section{How much Florida can be found in Lower Saxony? On the empirical evidence of the "creative class" in a German territorial Federal State}

DOI 10.1515/zfw-2016-0032

Eingereicht: 8. August 2016; akzeptiert: 22. Februar 2017;

online veröffentlicht: 7. März 2017

Zusammenfassung: Empirische Analysen zur Wirtschaftsgeographie der „Kreativen Klasse“ beschränken sich zumeist auf urbane Regionen. Der vorliegende Artikel testet drei von Richard Floridas Kernaussagen für das überwiegend nicht urban geprägte Bundesland Niedersachsen in Deutschland: die räumliche Verteilung der Mitglieder der „Kreativen Klasse“, ihr Binnenmigrationsmuster und ihre Gründungsmotive. Einige von Floridas Annahmen bestätigen sich auch für Niedersachsen, andere nicht. Politische Handlungsempfehlungen am Ende des Textes sollen helfen, die Wirkungen der Beschäftigungseffekte der „Kreativen Klasse“ auf die übrige Wirtschaft Niedersachsens zu steigern.

Schlüsselwörter: Entrepreneurship; Kreative Klasse; Kreativwirtschaft; Migration; Niedersachsen.

\begin{abstract}
Empirical results on the economic geography of the creative class are mainly restricted to urban areas. This paper tests three of Richard Florida's ideas for the largely non-urban Federal State of Lower Saxony, Germany: the spatial distribution of creative class members, their internal migration pattern and their entrepreneurial motivation. Some of Florida's assumptions hold true for Lower
\end{abstract}

\footnotetext{
*Korrespondierender Autor: Rolf Sternberg, Institute of Economic and Cultural Geography, Leibniz Universität Hannover, Schneiderberg 50, D-30167 Hannover, Germany, e-mail: sternberg@wigeo.uni-hannover.de

Christoph Alfken, Regionalverband Ruhr, Kronprinzenstraße 6, D-45128 Essen, Germany

Daniel Vossen, Institute of Economic and Cultural Geography, Leibniz Universität Hannover, Schneiderberg 50, D-30167 Hannover, Germany
}

Saxony as well, while others do not. Recommendations for policy-makers are provided in order to increase the effects of creative class employment on the real economy in Lower Saxony and its regions.

Keywords: creative class; creative industries; entrepreneurship; Lower Saxony; migration.

\section{Einführung}

Nach Hightech-Industrien in den 1970er und 1980er Jahren sowie Porters Clusterkonzept seit den 1990er Jahren ist Richard Floridas Idee der „Kreativen Klasse“ das gegenwärtige „Mantra“ (Sternberg 2012) insbesondere der kommunalen und regionalen Wirtschaftsförderung nicht nur in Deutschland. Trotz wechselnder Label - mal Kreativwirtschaft, mal Kulturwirtschaft, mal „Kreative Klasse" - bleibt die zentrale Botschaft gleich: Die Wirtschaftsförderung solle sich primär um die räumlich besonders mobilen, gründungsaffinen und spezifische Standorteigenschaften goutierenden Vertreter der „Kreativen Klasse" kümmern, denn sie würden aufgrund ihrer Merkmale die notwendigen ökonomischen Impulse generieren, ohne die Regionalwirtschaften heute nicht florieren könnten. Mit Florida (2004, S. 296): „I like to tell city leaders that finding ways to help support a local music scene can be just as important as investing in high-tech business and far more effective than building a downtown-mall”. Für Wirtschaftsförderer besonders attraktiv: eine Fokussierung auf die Anwerbung von Kreativen aus anderen Regionen bzw. die Bestandspflege bereits in der Region lebender Kreativer und ihrer Unternehmen ermögliche es auch strukturschwachen Kommunen und Regionen, sich mit realistischen Chancen auf den Hoffnungsträger Kreativ- 
wirtschaft zu konzentrieren, denn finanzielle Ressourcen seien nicht in großem Umfang erforderlich und die Karten im Regionenwettbewerb würden, so Florida, im Zeitalter der „Kreativen Klasse“ neu gemischt. Ergo zeigen zahlreiche Kultur- und Kreativwirtschaftsberichte von Städten, Kreisen und Regionen, aber auch von Bundesländern oder des Bundes von der hohen Popularität dieses Konzepts in Deutschland (vgl. z. B. Niedersächsische Ministerium für Wirtschaft und Verkehr/Niedersächsisches Ministerium für Wissenschaft und Kultur 2007; ICG 2011, Söndermann 2012). Diese Popularität scheint ungebrochen und betrifft die Wirtschaftsförderung und -politik auf allen räumlichen Maßstabsebenen. Exemplarisch sei auf der supranationalen Ebene die EU genannt, wo 2014 eine fraktionsübergreifende Arbeitsgruppe für Kultur- und Kreativwirtschaft geschaffen wurde, ein Jahr später der Europäische Rat die kulturellen und kreativen Crossover-Effekten bei der Förderung von Innovation, wirtschaftlicher Nachhaltigkeit und sozialer Inklusion betonte, das Programm „Creative Europe“ ins Leben gerufen wurde und die GD Binnenmarkt, Industrie, Unternehmertum und KMU förderte bis Ende 2014 die European Creative Industries Alliance (ECIA). Auch für das in diesem Beitrag untersuchte Bundesland Niedersachsen lässt sich die ungebrochene Aktualität des Themas auf Seiten der Politik gut dokumentieren (vgl. Niedersächsische Ministerium für Wirtschaft, Arbeit und Verkehr 2015 sowie http://www.mw.niedersachsen. de/startseite/themen/wirtschaft/dienstleistungen/kulturwirtschaft/kulturwirtschaft-15251.html). Diesbezüglich ist $\mathrm{zu}$ betonen, dass die „Kreative Klasse“ im Sinne Floridas weder mit der Kreativwirtschaft noch mit der Kulturwirtschaft identisch ist, weshalb auch in diesem Beitrag die drei Begriffe nicht synonym verwendet werden. Ähnlich wie zuvor beim Clusterkonzept hat die Wirtschaftspolitik schneller agiert als die empirische Forschung die Validität dieses Konzeptes zu prüfen in der Lage war. Ein in der akademischen Welt noch nicht hinreichend analysiertes, mindestens aber umstrittenes Konzept wurde und wird sehr zügig in die wirtschaftspolitische Praxis übertragen - mit dem Risiko der Fehlallokation öffentlicher Ressourcen in Zeiten begrenzter Mittel der öffentlichen Haushalte (also ein „mind-the-gap“-Problem im Sinne Kieses, vgl. Kiese 2008).

Floridas Konzept wurde und wird in Wirtschaft, Politik und Wissenschaft sehr unterschiedlich wahrgenommen. Der mehrheitlich sehr positiven Aufnahme in Wirtschaft und besonders der Politik steht teils harsche Kritik in der wirtschaftswissenschaftlichen (vgl. z. B. Glaeser 2005) und der wirtschaftsgeographischen Scientific Community (vgl. z. B. Markusen 2006; Peck 2005; Scott 2010; Storper/ Scott 2008; Sternberg 2012; Lange 2005, Krätke 2011) aus theoretischer und insbesondere aus empirischer Warte gegenüber. Für Wirtschaftsgeographen ist eine wissenschaftliche Auseinandersetzung mit dem Konzept auch deshalb interessant, weil es zahlreiche explizit wirtschaftsgeographische Bezüge besitzt. Exemplarisch seien genannt: die „Kreative Klasse“ ist, so Florida, räumlich hoch mobil, die Wanderungsintensität und -richtung wird von regionalen Merkmalen der Zielregion determiniert und ihre ökonomischen Effekte sind ebenfalls nicht raumneutral.

Der Beitrag möchte Forschungsfragen für Niedersachsen beantworten, die eng mit einigen der Kernaussagen von Floridas Konzept zusammenhängen. Auch wenn ein expliziter Test von Floridas Konzept nicht Ziel dieses Beitrages ist, orientiert er sich doch hinsichtlich zahlreicher terminologischer Aspekte an dessen Vorlage. Dies gilt explizit auch für die Definition der Kreativen selbst, die im Wesentlichen der berufsorientierten Begriffsabgrenzung der „Kreativen Klasse“ von Florida (2004) entspricht ( siehe Kap. 3 für empirische Details). Der Beitrag möchte in zweierlei Weise die Creative Class - Forschung bereichern und so einen Mehrwert für die empirische Wirtschaftsgeographie generieren. Erstens werden mit teils neuen Methoden und für diese Zwecke bislang nicht verwendeten Daten zwei Aspekte analysiert, die bislang - außer von Florida selbst - erstaunlicherweise kaum empirisch und regional differenziert untersucht wurden: die räumliche Mobilität Kreativer und deren Gründungsmotive. Zweitens geschieht dies für das Flächenland Niedersachsen, das in weiten Teilen nicht urban geprägt ist, sondern zum in Deutschland weit verbreiteten Typ der "Normalregion“ gehört. Die Untersuchung von Kreativen (auch) in ländlichen Regionen ist relevant, weil gemäß Floridas Thesen Kreative in urbanen Regionen absolut und relativ häufiger auftreten als in nicht-urbanen Regionen und ihre Beschäftigtenzahlen in städtischen Regionen auch stärker (bzw. überhaupt) an Beschäftigung zunehmen. Empirisch fundierte Analysen für nicht-urbane Räume fehlen aber, denn Florida selbst hat in den USA nur Metropolregionen untersucht und auch die sonstige empirische Forschung zur „Kreativen Klasse“ beschränkt sich entweder auf eine urbane Fallstudie (vgl. etwa Markusen 2006, Sailer/Papenheim 2007), auf mehrere Städte (z. B. Houston et al. 2008; Andersen et al. 2010b sowie das ACRE-Projekt; siehe http://www.acre.socsci.uva.nl/index.html) oder auf ganze Staaten. Selbst regional differenzierende empirische Studien unterscheiden i.d. R. nicht zwischen ländlichen und urbanen Regionen (für eine Ausnahme vgl. Mossig 2011). Zudem haben in den letzten Jahren auch zahlreiche nichturbane Regionen Niedersachsens (und anderer Bundesländer) wirtschaftspolitische Programme zugunsten von Kreativen aufgelegt (vgl. z. B. die zahlreichen Kultur- oder 
Kreativwirtschaftsberichte unter http://www.kulturwirtschaft.de/reports/). Die beiden genannten Ziele sind nur durch eine Kombination aus quantitativen und qualitativen Methoden und Daten zu erreichen (vgl. Kap. 3 zur Methodik und der Datengrundlage). Es ist explizit nicht das Ziel, das Konzept von Florida weiterzuentwickeln, sondern es auf einen bislang selten untersuchten, aber weit verbreiteten Regionstyp anzuwenden und mittels neuer Methoden und Daten empirisch zu prüfen.

Der Beitrag ist wie folgt gegliedert. Er beginnt in Kap. 2 mit einem knappen Überblick zum Stand der Kreativwirtschaftsforschung. Basierend auf den zuvor identifizierten (primär empirischen) Forschungslücken werden in Kap. 3 die Forschungsfragen formuliert, die Methodik erläutert und die Datengrundlage beschrieben. Im Kap. 4 beantworten die Verfasser die Forschungsfragen, ergänzt um die deskriptive Darstellung der regionalen Verbreitung der Kreativen im Untersuchungsraum. Kap. 5 widmet sich den wirtschaftspolitischen Schlussfolgerungen der zuvor dargestellten empirischen Befunde. Das abschließende Kap. 6 zieht (auch) ein selbstkritisches Fazit und skizziert Ideen zur zukünftigen Forschung.

\section{Floridas Konzept der „Kreativen Klasse" und seine wirtschafts- geographischen Implikationen: Stand der Forschung und offene Fragen}

Floridas Konzept ist vielfach dokumentiert (vgl. Florida 2002a,b; 2004, 2005a,b; 2008; Florida et al. 2008; Florida/Mellander 2010) und soll daher hier nur sehr knapp in Form von vier Kernaussagen wiedergegeben werden:

- Menschliche Kreativität (und nicht durch formale Bildung definiertes Humankapital) ist die zentrale und an Bedeutung gewinnende Quelle für volks- und regionalwirtschaftliches Wachstum

- Wirklich Kreative sind überdurchschnittlich gründungsaffin, räumlich sehr mobil und anspruchsvoll bzgl. ihrer Standortwahl („place matters“), wobei sie auf eher weiche Standortfaktoren (insbesondere Kultur, ein tolerantes und offenes Klima sowie von Menschen geschaffene „amenities“) als auf klassische ökonomische, also harte Faktoren ansprechen

- Die ökonomisch stärksten und innovativsten Regionen zeichnen sich durch besonders hohe Anteile beim
Creativity-Index aus (Modell der 3 Ts: Talent, Technology, Tolerance)

- Kreativität von Regionen lässt sich nicht allein über Technologie und Innovativität erfassen, sondern muss auch gesellschaftliche Offenheit und Toleranz (gegenüber ethnischen und anderen Minderheiten) berücksichtigen.

Forschungsstand und -lücken sind von einem Teil der Verfasser dieses Beitrages an anderer Stelle (vgl. Sternberg 2012) bereits dargestellt worden, weshalb hier lediglich eine Aktualisierung und explizite Bezugnahme auf Niedersachsen ergänzt wird. Die Zusammenfassung von Forschungsstand und -lücken orientiert sich an den im empirischen Teil (Kap.4) behandelten Forschungsfragen und übernimmt auch die dort gewählte Reihenfolge, beginnt ergo mit der regionalen Verteilung der „Kreativen Klasse“.

Komparative Aussagen zur regionalen Verteilung von Kreativen (und ihren ökonomischen Implikationen) sind nur bei vergleichbaren Definitionen von Kreativen sinnvoll. Schon in der wissenschaftlichen Literatur gibt es diesbezüglich wenig Konsens (vgl. zur Terminologiedebatte etwa Pratt 2005; für Deutschland Söndermann 2012 sowie BMWi 2009). Der vorliegende Beitrag verwendet im Wesentlichen die berufsorientierte Begriffsabgrenzung der „Kreativen Klasse“ Floridas (2004), auch wenn die Verfasser nicht meinen, es handele sich bei den Kreativen um eine Klasse im soziologischen Sinne (zu den Definitionsdetails vgl. Kap.3). Die Abgrenzung der „Kreativen Klasse“, insbesondere der Konflikt zwischen der eher kulturellen und der stärker ökonomischen Sichtweise von Kultur- bzw. Kreativwirtschaft (vgl. Uricchio 2004 sowie Lange/Bürkner 2010), bleibt aber eine Herausforderung für empirische Forschung.

Bei der empirischen Forschung zur regionalen Verteilung der Kreativen gewinnt die Operationalisierung der „Kreativen Klasse“ besondere Bedeutung (vgl. dazu auch die ausführliche Diskussion bei Alfken 2016, Kap.2). In Deutschland haben Fritsch/Stützer $(2007,2009)$ erstmals einen bundesweiten Versuch in diese Richtung gestartet. Sie grenzen kreative Berufe nach der ISCO-Klassifikation ab und verwenden dafür die Statistik der sozialversicherungspflichtig Beschäftigten (im Folgenden SvB). Für die in der SvB-Statistik der sozialversicherungspflichtig Beschäftigten nicht adäquat erfassten Künstler nutzen Fritsch und Stützer die Daten der Künstlersozialkasse. Selbstständige und Freelancer können nur partiell erfasst werden. Die Daten zeigen eine starke räumliche Konzentration der Kreativen insgesamt in urbanen Verdichtungsräumen, während in ländlichen Regionen gemäß BBSR-Klassifikation lediglich zehn Prozent der Kreativen 
lokalisiert sind. Die höchsten Anteile bei den Kreativen insgesamt weisen nicht die größten Städte, sondern mittelgroße Städte mit vielen und/oder großen Unternehmen in wissensintensiven Sektoren (z. B. Freiburg, Erlangen) auf. Bei den Künstlern und Freelancern ist das räumliche Muster deutlich disperser. Für Niedersachsen erlauben die Daten von Fritsch/Stützer (2007), basierend auf Zahlen von 2004, einen vorsichtigen Hinweis darauf, dass deren Fazit auch für Niedersachsen Gültigkeit haben könnte. Städte wie Göttingen und Osnabrück, zugleich Universitätsstandorte, weisen überproportional hohe Anteile der Kreativen an der Bevölkerung auf, was ebenfalls für die Stadt Hannover (nicht aber die Region Hannover) gilt. Mossig (2011) und Wedemeier (2015) zeigen bei etwas anderer Abgrenzung der „Kreativen Klasse“ und für BBSR-Regionstypen ähnliche Resultate zugunsten von Agglomerationsräumen und zuungunsten von ländlichen Regionen. Jüngst haben Alfken et al. (2015) eine flächendeckende Analyse für alle deutschen Kreise vorgelegt, in denen die Berufsgruppe der Künstler als ein wichtiger Teil der „Kreativen Klasse“ untersucht wird. Die bevölkerungsstarken Kreise liegen sowohl absolut als auch relativ (Künstler je Einwohner) an der Spitze, was den Besatz mit Künstlern betrifft. Die regionalen Unterschiede innerhalb der Gruppe der Künstler, differenziert nach vier Künstlersubgruppen, sind allerdings beträchtlich und entsprechen partiell nicht dem zuvor geschilderten Regionalmuster. Die bisher vorliegenden „Kulturwirtschaftsberichte“ für Niedersachsen (Niedersächsisches Ministerium für Wirtschaft, Technologie und Verkehr 2002; Niedersächsische Ministerium für Wirtschaft und Verkehr/Niedersächsisches Ministerium für Wissenschaft und Kultur 2007) sowie Söndermann (2014) geben sehr detaillierte Hinweise für einzelne Segmente des Kultur- und Kreativwirtschaftssektors, erlauben aber keinen flächendeckenden interregionalen Vergleich. Empirische Studien zur regionalen Verteilung von Kreativen außerhalb Deutschlands zeigen Parallelen, aber noch häufiger Unterschiede zum Muster in Deutschland (vgl. z. B. Vaarst et al. 2005 für Dänemark, Hansen/Niedomysl 2009 für Schweden, Boschma/Fritsch 2009). Für Niedersachsen fehlen derartige Analysen.

Hinsichtlich der räumlichen Mobilität Kreativer ist das Forschungsdefizit größer als für deren räumliche Verteilung, was primär Datenengpässe als Ursache hat. Dem Creative Class-Konzept zufolge sind Kreative interregional besonders mobil und stellen sehr spezifische Standortansprüche hinsichtlich weicher Standortfaktoren in der Zielregion. Lee et al. (2004) bestätigen dies für ausgewählte Metropolregionen in den USA, wofür es bislang für andere Länder oder gar deutsche Regionen kaum Belege gibt. Noch schwerer zu beantworten ist die Frage nach den De- terminanten der räumlichen Mobilität von Kreativen. Für Florida wandern die räumlich sehr mobilen Kreativen in Städte und Regionen, in denen bereits viele andere Kreative leben und ein tolerantes gesellschaftliches Klima (z. B. gegenüber Minderheiten) herrscht (vgl. dazu auch Brake 2015). Dies seien nicht nur Merkmale der Zielregionen, sondern auch die Motive der Migranten, in diese Regionen zu ziehen. Jüngere empirische Arbeiten zur räumlichen Mobilität von Hochqualifizierten in Europa zeigen diesbezüglich eher gemischte Resultate, mehrheitlich bestätigen sie die US-Befunde jedoch nicht (vgl. z. B. Möller/Tubadji 2009, Haisch/Klöpper 2007, Hansen/Niedomysl 2009, Wojan et al. 2007). Es sei aber, auch mit Bezug auf die Abgrenzungsdiskussion in Kap. 3, betont, dass Hochqualifizierte zwar eine Schnittmenge mit der „Kreativen Klasse“ aufweisen, aber weder mit ihr identisch noch eine Teilmenge von ihr sind. Gemäß der im empirischen Teil dieses Beitrags genutzten Daten der SvB der Bundesagentur für Arbeit zählen 2011 in Deutschland 4,6 Mio. Erwerbstätige zur „Kreativen Klasse“ und 3,1 Mio. zu den Hochqualifizierten mit Hochschulabschluss. Die Schnittmenge beider Populationen beträgt lediglich 1,7 Mio. Erwerbstätige.

Die meisten empirischen Arbeiten zu den Wanderungsmotiven Kreativer sind quantitativer Natur und argumentieren auf der Basis von Merkmalen der Zielregion der Migranten, ohne aber die tatsächliche, individuelle Motivation für die Standortwahl zu kennen. Nur wenige empirische Arbeiten adressieren dieses Thema direkt im Rahmen eines qualitativen Forschungsdesigns (vgl. Andersen et al. 2010a; Bennett 2010, Martin-Brelot et al. 2010), aber sie sind statisch, weil nur auf einen Zeitpunkt bezogen (für eine Ausnahme vgl. Borén/Young 2013). Offensichtlich genügt es nicht, allein auf regionaler Ebene aggregierte sozioökonomische Daten zu verwenden, um das Problem zu erfassen. Auch amtliche Sekundärdaten allein helfen womöglich nicht weiter, denn Wanderungsmotive sind dort nicht abgebildet. Die Analyse der Ursachen der Migration von Kreativen erfordert womöglich zum Teil andere Methoden der Datenerhebung, die es erlauben, die räumlichen und sozialen Trajektorien der Kreativen inkl. der dynamischen Dimension zu erfassen. Mindestens additiv sind qualitative Ansätze mit einem direkten Kontakt zu den Kreativen sinnvoll. In jedem Fall fehlen vergleichende, flächendeckende und empirisch fundierte Analysen zu Niedersachsen und wichtigen Teilräumen bzgl. der Mobilität von Kreativen, was auch für Deutschland insgesamt gilt.

Abschließend sei auf die wichtige Frage des Potenzials der „Kreativen Klasse“ hingewiesen: sie muss rein quantitativ groß genug sein, um überhaupt signifikante Wachstumseffekte auf regionaler Ebene auslösen zu kön- 
nen. Dies hängt unmittelbar von der Definition der „Kreativen Klasse“ ab: bei Florida umfasst sie 30\% der Beschäftigten in den von ihm untersuchten Metropolregionen. Kreativwirtschaftsberichte zu europäischen Ländern oder Städten mit einer engeren Abgrenzung kreativer Branchen und/oder Berufe kommen dagegen auf deutlich geringere Anteile unter zehn Prozent (vgl. Deutscher Bundestag 2007, BMWi 2009). Für den vorliegenden Beitrag stellt sich daher auch die Frage, wie groß (absolut) die „Kreative Klasse“ in Niedersachsen und ihren Teilräumen tatsächlich ist.

Eine spezifische (regional-) ökonomische Wirkung von Kreativen entfaltet sich über deren, so Florida (2005), überproportionale Gründungsaktivität (vgl. auch Lee et al. 2004). Dieser Teil der Hypothesen Floridas ist bislang empirisch für europäische oder gar deutsche Regionen kaum geprüft. Eine Ausnahme stellt die Arbeit von Fritsch/ Sorgner (2014) dar. Mittels einer Stichprobe des Sozioökonomischen Panels (SOEP) erklären sie auf Basis deutscher Regionen die Gründungsaktivität unter Kreativen in Deutschland. Sie folgen Floridas (2004) Ansatz und klassifizieren Personen in die Berufsgruppen „creative core” (Hochkreative, z. B. Berufe wie Wissenschaftler, Informatiker, Ingenieure oder Architekten), „creative professionals“ (z. B. Unternehmensberater, Juristen, Bankfachleute oder technische Fachkräfte), und „noncreative professions”, die auf der International Classification of Occupations (ISCO-88) beruht. Gut ein Drittel des SOEP-Samples gehört zur „Kreativen Klasse“; das Sample besteht also zum größeren Teil aus Befragten in nicht-kreativen Berufen. Die Autoren zeigen erstens, dass Kreative tatsächlich häufiger Gründer von Unternehmen sind als dies bei nicht zur „Kreativen Klasse“ Gehörenden der Fall ist, und dass, zweitens, in Regionen mit besonders hohem Anteil Kreativer diese auch überproportional häufig selbstständig erwerbstätig sind. Von den genannten Studien abgesehen, ist die Forschungslücke bzgl. Gründungsaktivitäten der „Kreativen Klasse“ eklatant. Zwar mehren sich in jüngerer Zeit Artikel (vgl. z. B. Sundbo 2011, Ward 2004) und auch Sammelbände zum Zusammenhang von Entrepreneurship und Kreativität (vgl. z. B. Sternberg/Krauss 2014, Book/Phillips 2013, McMullan/Kenworthy 2015), aber das regional differenzierende Moment fehlt in diesen Arbeiten zumeist, während kognitive Aspekte und Persönlichkeitsmerkmale im Mittelpunkt stehen. Für Niedersachsens Regionen existieren keinerlei empirische Befunde zu diesem Thema.

\section{Forschungsfragen, Datengrund- lage und Methodik}

Ziel des Beitrages sind primär empirische Antworten auf die folgenden Forschungsfragen, jeweils bezogen auf das eher ländlich geprägte Flächenland Niedersachsen:

- wie ist die „Kreative Klasse“ räumlich verteilt?

- welche Grundmuster, Dynamiken und Erklärungen lassen sich für die räumliche Mobilität Kreativer erkennen?

- welche Gründungsmotive kennzeichnen Kreative?

Für einen empirischen Beitrag mit intendierter Anlehnung an das Konzept Floridas ist die Definition Kreativer, der „Kreativen Klasse“ bzw. der Kreativwirtschaft von erheblicher Bedeutung. In der Regel basiert das Paper auf Floridas berufsorientierter Abgrenzung. Für die quantitativen Untersuchungen (betreffen mehrheitlich die ersten zwei Forschungsfragen) wird die „Kreative Klasse“ in Anlehnung an Vorarbeiten des Instituts für Arbeitsmarkt- und Berufsforschung (IAB) anhand von 45 kreativen Berufen der Klassifikation der Berufe (KldB 1988) definiert. Diese Definition ist identisch mit jener von Möller/Tubadji (2009) und zählt nach eigenen Berechnungen auf Basis von Daten der Bundesagentur für Arbeit (BA 2012) für das Jahr 2011 in Deutschland 4,6 Mio. SvB zu den Kreativen. Dies entspricht 16,3\% aller SvB und damit deutlich weniger als bei Florida, der bei seiner weiteren Definition auf fast ein Drittel der Beschäftigung kommt, was in der Literatur stark kritisiert wird, vgl. z. B. Chantelot et al. (2010). Ähnlich wie bei Florida wird die „Kreative Klasse“ hierbei in drei Gruppen unterteilt. Auf die Hochkreativen („super creative core“) entfallen 2,3 Mio. SvB oder 8,2\% sowie 18 Berufe der BA-Klassifikation. Zur zweiten Gruppe der „creative professionals“ zählen 2,0 Mio. SvB oder 7,2\% sowie 19 Berufe. Die dritte und kleinste Gruppe der „Kreativen Klasse“, gleichzeitig eine Subgruppe der Hochkreativen, bilden die „Bohemiens“ (253 Tsd. SvB, 0,9\% aller SbV; 8 Berufe), u. a. mit Schriftstellern, Musikern, bildenden und darstellenden Künstlern sowie Designern.

Den Autoren ist bewusst, dass auch die hier gewählte Abgrenzung nicht frei von Schwächen ist. Manche der von Higgs/Cunningham (2008) und Glückler et al. (2010) zurecht angesprochenen Probleme, sich zwischen einer funktionalen, auf Berufe ausgerichteten und einer sektoralen (Branchen) Perspektive entscheiden zu müssen, lassen sich auch durch das hier gewählte Verfahren nicht vermeiden. Zumindest haben wir aber die Breite und Heterogenität der „Kreativen Klasse“ reduziert und damit das wichtigste Argument der Kritiker der Abgrenzung Floridas 
berücksichtigt, die eine Reduzierung der Anzahl der berücksichtigten Berufe - insbesondere um solche, die allein standardisierte Dienstleistungen bereitstellen, ohne explizit kreativ zu sein - fordern (vgl. Markusen 2006, Comunian/Faggian/Li 2010, McGranahan/Wojan 2007, Marlet/van Woerkens 2007).

Die qualitativen Daten aus einer der beiden primärstatistischen Erhebungen (ein Teil der zweiten sowie die dritte Forschungsfrage) adressieren Gründer und Unternehmer aus der Design- und Werbebranche. Letztere weisen sowohl eine hohe Schnittmenge mit der in Deutschland üblichen Abgrenzung der Kultur- und Kreativwirtschaft als auch mit der „Kreativen Klasse“ Floridas auf, insbesondere auch mit der Subgruppe der Bohemiens. Zudem umfasst die Design- und Werbebranche mit mehr als 30\% der Unternehmen, der Beschäftigten und des Umsatzes den größten Teil der deutschen Kultur- und Kreativwirtschaft (vgl. BMWi 2009).

Die quantitativen Daten zur Verteilung und zum Wachstum der „Kreativen Klasse“ in den Kreisen und Regionen Niedersachsens wurden anhand einer Sonderauswertung der SvB-Statistik nach Berufsgruppen der Bundesagentur für Arbeit ermittelt (im Folgenden: BA 2014). Darüber hinaus wurde die schwach anonymisierte Stichprobe der Integrierten Arbeitsmarktbiografien (SIAB 1975-2010) am Forschungsdatenzentrum der Bundesagentur für Arbeit im IAB (im Folgenden: SIAB 2014) genutzt, um die Migrationsraten auf Ebene der Raumordnungsregionen zu berechnen. Weitere amtliche und nicht-amtliche Sekundärstatistiken dienten als potenzielle Determinanten der Verteilung, Entwicklung und Migration der „Kreativen Klasse“ auf der Makroebene.

Zusätzlich zur amtlichen Statistik wurden 2013 eigene Erhebungen in drei Normalregionen (Hannover, Osnabrück und Göttingen) durchgeführt, um die individuellen Migrationsmotive zu ermitteln. Mit Hilfe der lokalen Wirtschaftsförderungen und ergänzenden Onlinerecherchen (u. a. Unternehmensregister, Branchennetzwerke, Mitgliederlisten von Kammern und Verbänden, Soziale Netzwerke) wurden 818 Unternehmensadressen recherchiert und im Rahmen einer Onlinebefragung angeschrieben. 244 Angeschriebene beteiligten sich an dieser Befragung (30\% Rücklauf). Diese Onlinebefragung diente dazu, einen generellen Überblick über das Ausmaß und die Motive der Migration zu erhalten und potenzielle Interviewpartner für den nachfolgend skizzierten Erhebungsschritt zu identifizieren.

Zwischen Juli und November 2013 wurden 58 leitfadengestützte, halbstrukturierte face-to-face Interviews mit Kreativen der Werbe- und Designbranche in den genannten Regionen von 40-150 Minuten Länge geführt.
Ziel dieser Interviews war es, das Migrationsverhalten einzelner Individuen aus zwei eng und eindeutig abgegrenzten Branchen der „Kreativen Klasse“ über deren gesamten Lebensverlauf detailliert zu erfassen. 36 Interviews fanden in Hannover, 15 in Osnabrück und 7 in Göttingen statt. Gesprächspartner waren sowohl Beschäftigte als auch Unternehmer (meistens Gründer) aus der Werbe- bzw. Designerbranche. Sie wurden nach dem Zufallsprinzip aus den oben erwähnten 244 an der Online-Befragung Beteiligten ausgewählt. Zur Identifizierung von Karrierepfaden und räumlicher Mobilität der Befragten wurde die Life History Calendar Methode angewendet, die die Nutzung retrospektiver Daten ermöglicht, ohne die Nachteile verzerrter Wahrnehmung in Kauf nehmen zu müssen (vgl. Freedman et al. 1988; Harris/Parisi 2007). Der (kurze) standardisierte Teil der Befragung wurde später quantitativ ausgewertet. Der qualitative Teil der Befragung wurde, nach digitaler Aufzeichnung und Transkribieren aller Interviews, mit Hilfe des Programms NVivo 9 einer qualitativen Inhaltsanalyse unterzogen. Das finale Kodierungsschema unterscheidet sieben Gründungsmotive und ist auf Nachfrage bei den Autoren erhältlich. Drei Themen standen im Mittelpunkt dieser Erhebung: der Verlauf der beruflichen Karriere, die interregionale Mobilität sowie, im Falle einer Gründerperson, deren Gründungsmotiv(e) und die ökonomischen Kennzahlen der Gründung im Zeitablauf. Für weitere Details zu diesen qualitativen Daten vgl. Alfken (2015).

Methodisch wechseln im Beitrag quantitative und qualitative Ansätze. Die absolute und relative räumliche Verteilung, das Wachstum und die Migration der „Kreativen Klasse“ in Niedersachsen und seinen Regionen werden durch deskriptive Auswertungen und Darstellungen quantitativ untersucht. Regressionsanalysen dienen der Analyse der Determinanten der Migration, der regionalen Verteilung und dem regionalen Wachstum der „Kreativen Klasse“. Die Daten der Onlinebefragung wurden zusätzlich deskriptiv ausgewertet, um das Ausmaß und die Motive sowie die Herkunft und Zielregion der Migration der kreativen Gründer und Unternehmer der Design- und Werbebranche in den drei Teilregionen zu beschreiben. Mit Hilfe von qualitativen Inhaltsanalysen wurde schließlich das Migrationsverhalten der Interviewten im Detail analysiert. 


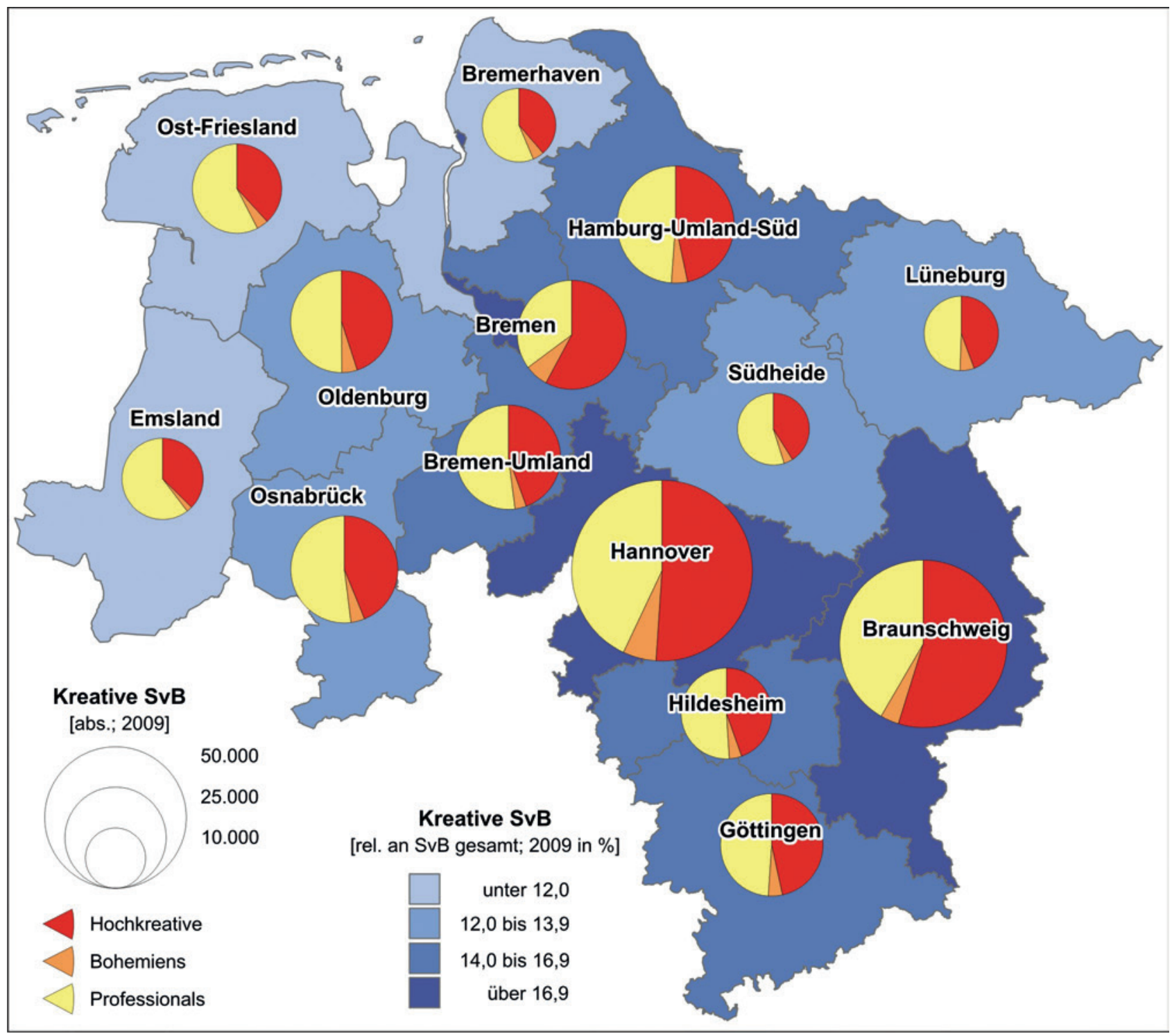

Abbildung 1: Räumliche Verteilung der „Kreativen Klasse“ in Niedersachsens Raumordnungsregionen 2009. Quelle: BA 2014; eigene Berechnungen und Darstellung.

\section{Empirische Ergebnisse}

\subsection{Zur regionalen Verteilung der Kreativen in Niedersachsen}

Floridas Thesen lassen erwarten, dass in urbanen Regionen Kreative absolut und relativ häufiger auftreten als in nicht-urbanen Regionen und dass Kreative in städtischen Regionen auch stärker (bzw. überhaupt) an Beschäftigung zunehmen.

Abbildung 1 illustriert den Anteil der „Kreativen Klasse“ an allen SvB sowie die absolute Anzahl kreativer SvB unterteilt nach Subgruppen der „Kreativen Klasse“ in den niedersächsischen Raumordnungsregionen. Insgesamt waren im Jahr 2009 knapp 378.000 Kreative in Niedersachsen beschäftigt; der 14,8\%-Anteil an den SvB liegt leicht unter dem Wert Deutschlands insgesamt (16,4\%). Niedersachsen liegt damit im Bundesländervergleich deutlich vor allen ostdeutschen Flächenländern, dem Saarland und Schleswig-Holstein, aber hinter den übrigen Ländern. Die größte Subgruppe der niedersächsischen Kreativen bilden mit fast 183.000 Personen (48,4\%) die Professionals, gefolgt von den Hochkreativen (178.000, ohne Bohemiens) und den Bohemiens (17.000). 
Tabelle 1: Sozialversicherungspflichtig Beschäftigte der „Kreativen Klasse“ nach siedlungsstrukturellen Regions- und Kreistypen 2009 bzw. 1999-2009. Quelle: BA 2014; eigene Berechnungen und Darstellung.

\begin{tabular}{|c|c|c|c|c|c|c|}
\hline \multirow{2}{*}{$\begin{array}{l}\text { Siedlungsstrukturelle Regions-/ } \\
\text { Kreistypen des Bundesinstituts für } \\
\text { Bau-, Stadt- und Raumforschung } \\
\text { (BBSR) }\end{array}$} & \multicolumn{3}{|c|}{ Niedersachsen } & \multicolumn{3}{|c|}{ Deutschland } \\
\hline & $\begin{array}{r}\text { Absolut } \\
(1.000 \\
\text { SvB })\end{array}$ & $\begin{array}{r}\text { Relativ } \\
\text { (\% der } \\
\text { SvB) }\end{array}$ & $\begin{array}{r}\text { Mittleres jährliches } \\
\text { Wachstum 1999- } \\
2009(\%)\end{array}$ & $\begin{array}{r}\text { Absolut } \\
(1.000 \\
\text { SvB })\end{array}$ & $\begin{array}{r}\text { Relativ } \\
(\% \text { der } \\
\text { SvB })\end{array}$ & $\begin{array}{l}\text { Mittleres jährli- } \\
\text { ches Wachstum } \\
\text { 1999-2009 (\%) }\end{array}$ \\
\hline $\begin{array}{l}\text { Regionstypen insgesamt (13 bzw. } \\
\text { 96) }\end{array}$ & 380,9 & 14,77 & 0,80 & $4.481,9$ & 16,44 & 0,74 \\
\hline $\begin{array}{l}\text { Städtische Raumordnungsregionen } \\
\text { (1 bzw. 24) }\end{array}$ & 79,5 & 17,34 & 0,53 & $2.427,6$ & 19,23 & 0,77 \\
\hline $\begin{array}{l}\text { ROR mit Verstädterungsansätzen ( } 8 \\
\text { bzw. } 35 \text { ) }\end{array}$ & 225,9 & 14,32 & 0,87 & $1.260,1$ & 14,87 & 0,85 \\
\hline $\begin{array}{l}\text { Ländliche Raumordnungsregionen } \\
\text { (4 bzw. 37) }\end{array}$ & 75,5 & 13,92 & 0,87 & 794,2 & 12,88 & 0,49 \\
\hline Kreistypen insgesamt (46 bzw. 412) & 377,8 & 14,84 & 0,81 & $4.481,9$ & 16,44 & 0,74 \\
\hline Kreisfreie Großstädte (5 bzw. 68) & 49,5 & 19,23 & 1,53 & $1.495,6$ & 20,04 & 1,08 \\
\hline Städtische Kreise (11 bzw. 138) & 148,8 & 16,71 & 0,56 & $1.864,0$ & 17,04 & 0,56 \\
\hline $\begin{array}{l}\text { Ländliche Kreise mit Verdichtungs- } \\
\text { ansätze (14 bzw. 100) }\end{array}$ & 90,2 & 13,31 & 0,74 & 636,8 & 13,67 & 0,66 \\
\hline $\begin{array}{l}\text { Dünn besiedelte ländliche Kreise } \\
\text { (16 bzw. 106) }\end{array}$ & 89,4 & 12,41 & 0,91 & 485,5 & 11,56 & 0,51 \\
\hline
\end{tabular}

SvB, Sozialversicherungspflichtig Beschäftigte.

Die Raumordnungsregionen (RORs) Hannover und Braunschweig liegen mit knapp 80.000 bzw. über 68.000 Kreativen absolut, aber auch relativ vor den übrigen niedersächsischen, nicht urbanen RORs, insbesondere dem ländlichen Nordwesten Niedersachsens mit den RORs OstFriesland (10,4\% Anteil der Kreativen an den SvB), Bremerhaven (mit den Landkreisen Cuxhaven und Wesermarsch; 10,6\%) und dem Emsland (11,5\%). Ein sehr ähnliches Bild zeigt sich auf der Kreisebene. Insbesondere in den größeren kreisfreien Städten wie Braunschweig (23,5\%), Wolfsburg (19,9\%), Oldenburg (19,0\%) und Osnabrück (16,5\%) zeigen sich überdurchschnittliche Konzentrationen kreativer SvB. Auch die „Region Hannover“ (umfasst den früheren Landkreis Hannover sowie die Stadt Hannover) reiht sich hier ein $(18,3 \%)$. Weit unterdurchschnittliche Anteile sind in den Kreisen im Nordwesten, entlang der Ems-Achse, und in Südniedersachsen (ohne Göttingen) zu beobachten.

Wie die folgende Tabelle, aber auch die Regressionsmodelle in Tabelle 3 (Kap.4.2) nahelegen, ist für dieses Regionalmuster ein Mix aus harten und weichen Standortfaktoren verantwortlich. Bei der regionalen Verteilung des Anteils der sozialversicherungspflichtig beschäftigten Mitglieder der „Kreativen Klasse“ in Niedersachsen spielen Arbeitsmarktaspekte (z.B. Lohnniveau; positiv korreliert mit dem Anteil an beschäftigten Kreativen) und Lebenshaltungskosten (negativer Zusammenhang) ebenso eine wichtige Rolle wie etwa die kulturelle Viel- falt (positiver Zshg.). Die hohen Anteile in der Raumordnungsregion Braunschweig sind insbesondere auf die zahlreichen Ingenieure (die zu den „Hochkreativen“ gehören, vgl. Abbildung 1) in der Automotive-Wirtschaft in Wolfsburg zurückzuführen. Tabelle 1 und damit eine rein statische Betrachtung bestätigt Floridas Thesen ebenso wie die Analysen von Fritsch/Stützer $(2007,2009)$ für Deutschlands Teilräume, denn hohe Anteile kreativer Beschäftigter finden sich insbesondere in Regionen mit Universitäten und/oder urbanen Regionen wie Hannover, Braunschweig und Bremen, die auch hohe Werte bei Toleranz und Offenheit aufweisen. In den meisten ländlich geprägten Raumordnungsregionen Niedersachsens dagegen sind die Werte für Offenheit und Toleranz geringer, was deren aktuell relativ geringen Anteil an kreativen Beschäftigten plausibel erscheinen lässt. Die Interpretation von Tabelle 3 (Kap. 4.2) liefert ausführlichere Erklärungen der statischen Verteilung sowie der zeitlichen Veränderung der Anteile der kreativen Beschäftigung in den Regionen Niedersachsens.

Wie nach den bisherigen Befunden zu erwarten, steigt für die aggregierten siedlungsstrukturellen Regions- und Kreistypen gemäß BBSR (Gebietsstand 2009) der Anteil der kreativen SvB kontinuierlich mit zunehmendem Grad der Verstädterung bzw. Zentralität und Verdichtung der Raumeinheit an (vgl. Tabelle 1). Kreative finden sich demzufolge absolut und relativ vermehrt in urbanen Räumen. Diese Resultate der statischen Analyse entsprechen den 


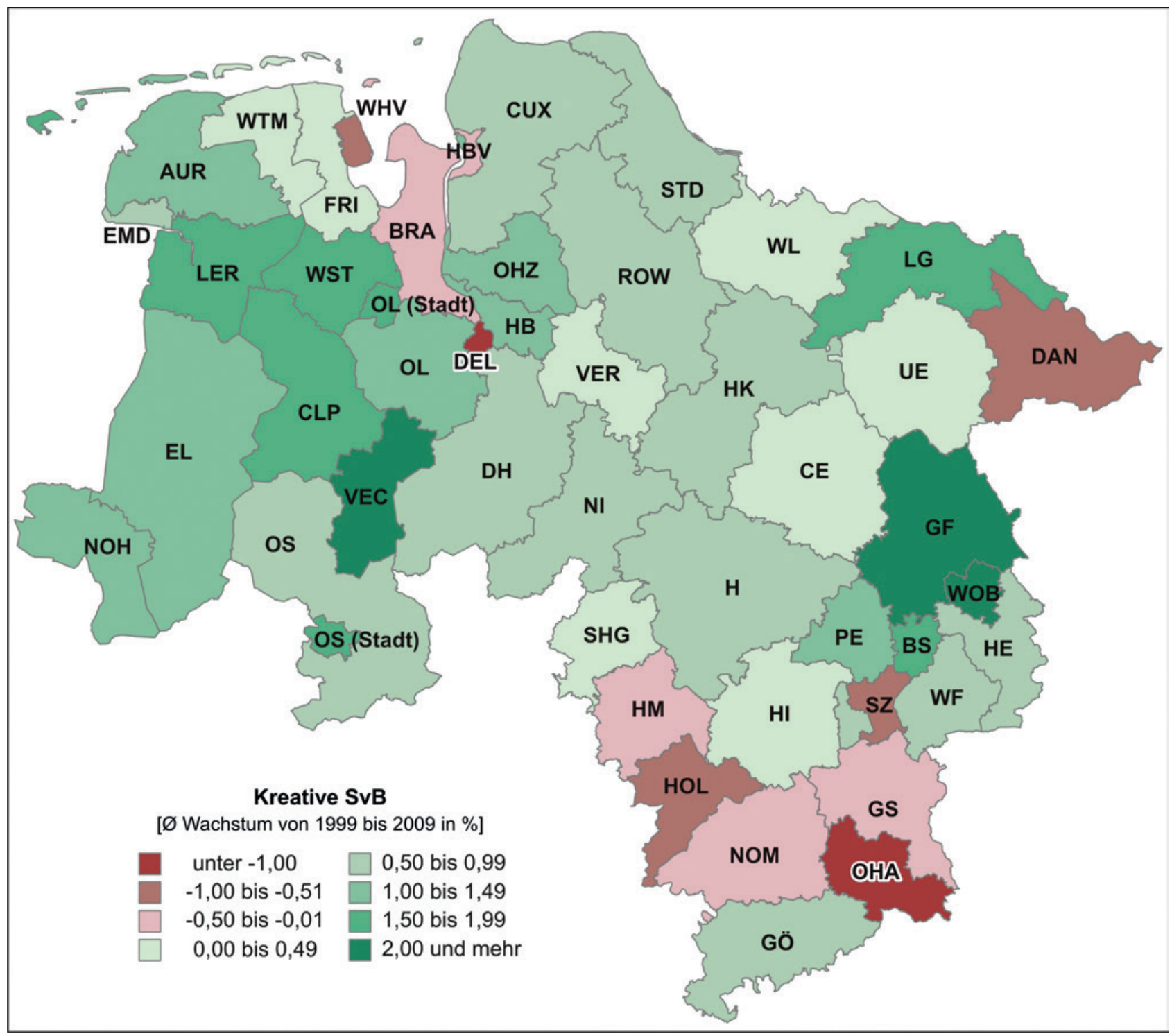

Abbildung 2: Beschäftigungsentwicklung der „Kreativen Klasse“ in Niedersachsens Kreisen und kreisfreien Städten 1999-2009. Quelle: BA 2014; eigene Berechnungen und Darstellung.

gemäß Florida zu erwartenden. Ob allerdings tatsächlich eher weiche Faktoren wie die kulturelle Vielfalt oder das von Florida postulierte Klima der Toleranz und Offenheit ursächlich sind, wird in Kap.4.2 analysiert.

Bei der Betrachtung der dynamischen Perspektive wandelt sich das Regionalmuster. Im Zeitraum 1999 bis 2009 sind die SvB der „Kreativen Klasse“ Niedersachsens absolut knapp 30.000 SvB gewachsen, was einem Anteil von $0,81 \%$ entspricht. Damit liegt Niedersachsen über dem Bundesdurchschnitt (vgl. Tabelle 1) und auf dem dritten Rang unter den Flächenländern. Hierfür verantwortlich ist insbesondere die überdurchschnittliche Zunahme der Hochkreativen $(+1,9 \%)$ und der Bohemiens $(+1,4 \%)$.
Abbildung 2 zeigt sehr unterschiedliche Entwicklungen für die Stadt- und Landkreise. Unter den Kreisen mit den größten relativen Zuwächsen finden sich solche sowohl in urbanen als auch solche in ländlichen RORs (z. B. $+2,7 \%$ für die Stadt Wolfsburg, $+2,0 \%$ für den Nachbarlandkreis Gifhorn, $+2,2 \%$ für Vechta, $+1,9 \%$ für Cloppenburg, $+1,6 \%$ für die Stadt Oldenburg). Andere Stadtkreise wie Salzgitter, Delmenhorst und Wilhelmshaven, aber auch große Teile Südniedersachsens sind dagegen durch eine Abnahme kreativer Beschäftigung im Beobachtungszeitraum gekennzeichnet. Den größten absoluten Zuwachs verzeichnet mit knapp 3.800 SvB die Region Hannover (+0,6\% im Jahresmittel). Ein entsprechendes Bild zeigt sich auf der Ebene der Raumordnungsregionen, 


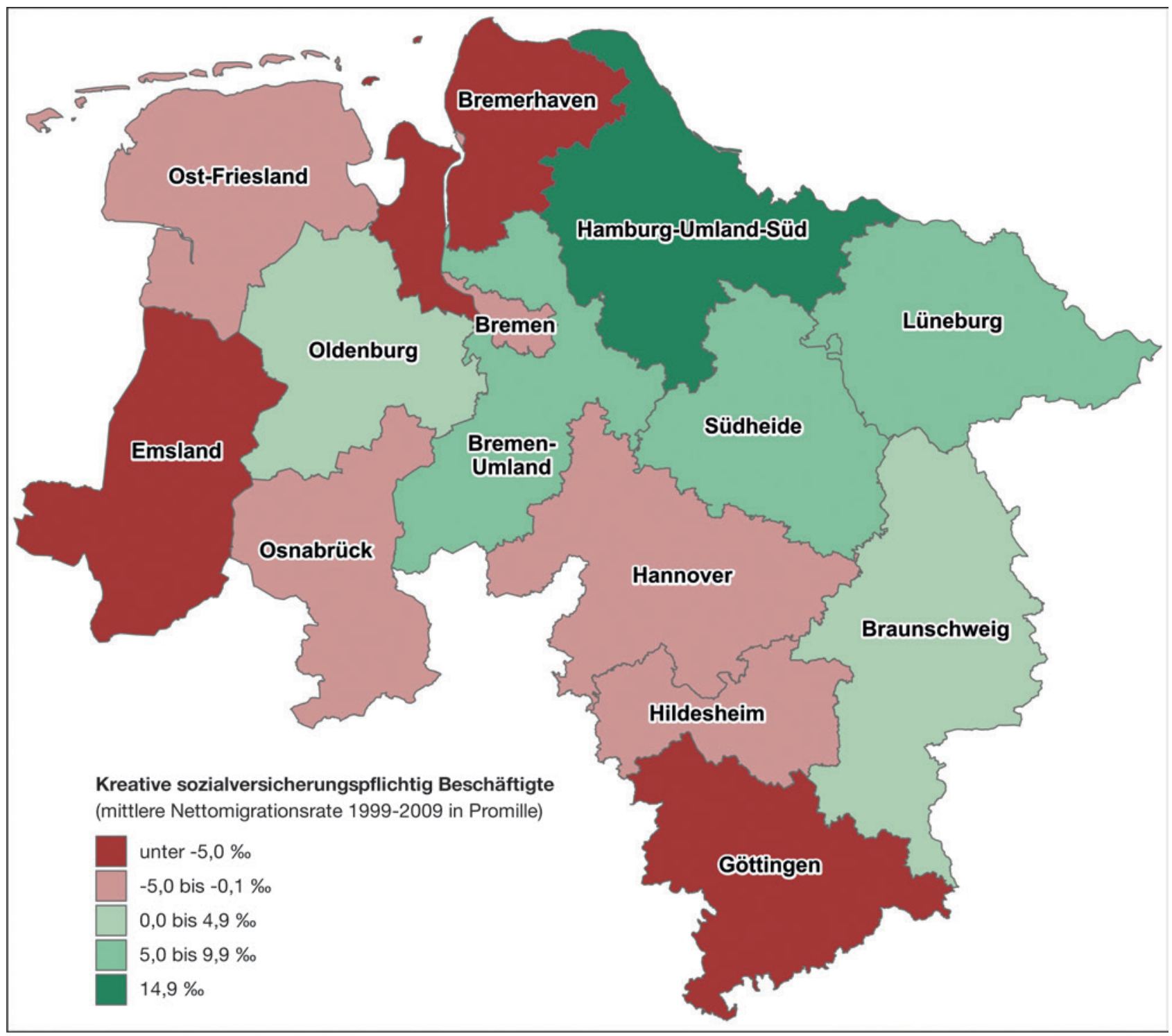

Abbildung 3: Nettomigrationsraten der „Kreativen Klasse“ in Niedersachsens Raumordnungsregionen 1999-2009. Quelle: SIAB 2014; eigene Berechnungen und Darstellung.

wobei die Region Braunschweig mit fast 7.900 SvB absolut am stärksten wuchs.

Innerhalb Niedersachsens zeigen die Daten für den Beobachtungszeitraum Hinweise auf eine regional konvergente Entwicklung bei den SvB der „Kreativen Klasse“, was nicht mit Floridas Thesen übereinstimmt. Dies ist nicht allein durch den Basiseffekt zu erklären, von dem kleinere Regionen mit geringeren Werten zum Ausgangszeitpunkt bei dynamischer Perspektive oft profitieren. Das relative Wachstum der „Kreativen Klasse“ nimmt mit zunehmendem Grad der Verstädterung bzw. Zentralität und Verdichtung der Raumeinheit in Niedersachsen ab (vgl. Tabelle 1). Dies gilt jedoch nicht für die „Kreisfreien Großstädte“, die 1999-2009 weit überdurchschnittlich gewach- sen sind. Da auch Niedersachsen insgesamt, ebenso wie manche ländliche Räume (z. B. das westliche Oldenburger Land), überdurchschnittliche Wachstumsraten aufweisen, deutet dies auf einen langsamen Aufholprozess bis dato weniger kreativer Räume innerhalb Niedersachsens hin, wohingegen Südniedersachsen und die JadeBay Region (weiter) an Boden verloren haben.

\subsection{Das Migrationsverhalten der Kreativen: Umfang, Motive und Zielregionen}

Florida geht von einer überdurchschnittlich hohen räumlichen Mobilität Kreativer aus, die zudem spezifische Mo- 
Tabelle 2: Migrationsbilanz der „Kreativen Klasse“ in Niedersachsen 1999-2009. Quelle: SIAB 2014; eigene Berechnungen und Darstellung.

\begin{tabular}{|c|c|c|c|}
\hline $\begin{array}{l}\text { Niedersächsische Raumordnungs- } \\
\text { regionen }\end{array}$ & $\begin{array}{r}\text { Mittlere jährliche } \\
\text { Zuwanderungsrate (\%o** }\end{array}$ & $\begin{array}{r}\text { Mittlere jährliche } \\
\text { Abwanderungsrate (\%o*** }\end{array}$ & $\begin{array}{r}\text { Mittlere jährliche } \\
\text { Nettomigrationsrate (\%o)*** }\end{array}$ \\
\hline \multicolumn{4}{|l|}{ Städtische Raumordnungsregionen } \\
\hline Hannover & 24,2 & 27,6 & $-3,4$ \\
\hline \multicolumn{4}{|l|}{ ROR mit Verstädterungsansätzen } \\
\hline Bremen-Umland & 38,0 & 28,9 & 9,1 \\
\hline Oldenburg & 34,0 & 31,5 & 2,5 \\
\hline Braunschweig & 23,5 & 23,1 & 0,4 \\
\hline Ost-Friesland & 20,5 & 24,0 & $-3,5$ \\
\hline Hildesheim & 32,7 & 37,0 & $-4,2$ \\
\hline Osnabrück & 25,6 & 30,5 & $-4,9$ \\
\hline Bremerhaven & 23,3 & 29,0 & $-5,7$ \\
\hline Göttingen & 24,8 & 33,5 & $-8,7$ \\
\hline \multicolumn{4}{|l|}{ Ländliche Raumordnungsregionen } \\
\hline HH-Umland-Süd & 44,4 & 29,5 & 14,9 \\
\hline Südheide & 33,7 & 24,6 & 9,1 \\
\hline Lüneburg & 34,8 & 27,2 & 7,6 \\
\hline Emsland & 18,3 & 24,3 & $-6,1$ \\
\hline
\end{tabular}

* Zuwanderer / Bestand * 1000; ** Abwanderer / Bestand * 1000; ** Zuwanderungsrate - Abwanderungsrate.

tive und Attribute der Zielregionen kennzeichnen. Die mittels der SIAB berechneten Wanderungsraten nach Berufsgruppen zeigen, zunächst für Deutschland insgesamt, ein ambivalentes Bild. Im Zeitraum von 1999 bis 2009 sind pro Jahr durchschnittlich ca. 90.000 Kreative von einer in eine andere Raumordnungsregion gewandert. Gewichtet mit dem Bestand an kreativen SvB ergibt sich eine Wanderungsquote von $27,1 \%$ (also 27 von 1.000 Kreativen). Damit sind Kreative mobiler als die übrigen Beschäftigten mit einer Quote von 17,9\%o. Zwischen den drei Subgruppen der Kreativen bestehen deutliche Unterschiede. Während die Bohemiens $(41,2 \%$ ) und die übrigen Hochkreativen $(32,2 \%$ ) räumlich sehr mobil sind, bewegen sich die Kreativen Professionals mit 20,4\%o auf dem Niveau der SvB insgesamt (19,6\%).

Wichtiger für die regionalökonomische Wirkung der Mobilität der „Kreativen Klasse“ sind die Wanderungsrichtung sowie die besonders attraktiven Zielregionen. Letztere lassen sich über die Nettomigrationsraten approximieren, die in Abbildung 3 als Jahresmittelwerte für den Zeitraum 1999 bis 2009 und auf Ebene der niedersächsischen Raumordnungsregionen dargestellt sind.

Insbesondere die Umlandregionen der großen Zentren sind durch eine hohe Nettozuwanderung gekennzeichnet. An der Spitze steht die ROR Hamburg-Umland-Süd mit einer Nettozuwanderung von jährlich fast 15 je 1.000 Kreative (absoluter Zuwachs von ca. 4.000 SvB im genannten Zeitraum). Ebenfalls von Abwanderungen aus den Großstädten profitieren andere RORs im erweiterten Hamburger Umland (Südheide $+9,3 \%$; Lüneburg $+7,6 \%$ ) sowie das Bremer Umland (+9,1\%o). Auch Oldenburg (2,5\%o) und Braunschweig $(0,4 \%$ ) haben netto Kreative dazugewonnen, während Südniedersachsen (Göttingen: -8,7\%o) und die JadeBay Region (ROR Bremerhaven: -5,7\%0) sowie das Emsland $(-6,1 \%$ ) Wanderungsverluste hinnehmen mussten. Auch Regionen mit großen Zentren wie Hannover und Osnabrück gehören zu den Nettoabwanderungsgebieten (vgl. Tabelle 2).

Für Florida sind spezifische Attribute der Zielregionen der Kreativen dafür verantwortlich, dass und wohin Kreative wandern. Im Folgenden wird daher geprüft, ob es einen Zusammenhang zwischen den geschilderten Nettomigrationsraten, dem Bestand an Kreativen (Anteil an den SvB) sowie deren Entwicklung im Zeitablauf einerseits und den - weichen oder harten - Standortfaktoren dieser Regionen andererseits besteht. Wegen der nur 13 Raumordnungsregionen in Niedersachsen und fehlender Werte für die Kreisebene, erfolgen die multivariaten Regressionsmodelle auf der aggregierten Ebene aller 96 deutschen Raumordnungsregionen.

Um den diversen Facetten regionaler Standortfaktoren - wie z.B. den Arbeitsmarktbedingungen gerecht zu werden - werden jeweils mehrere Indikatoren ${ }^{1}$ mittels Hauptkomponentenanalyse $\mathrm{zu}$ einem übergeordneten

1 Je nach Datenverfügbarkeit sind die einfließenden Variablen den regionalen Zielgrößen zeitlich vorgelagert. Die Resultate der Hauptkomponentenanalyse sowie die Liste sämtlicher Ausgangsvariablen sind bei den Autoren auf Anfrage erhältlich; ihre Integration in den Beitrag hätte dessen quantitativen Rahmen gesprengt. 
Tabelle 3: Determinanten des Beschäftigtenanteils, des Wachstums und der Migration der „Kreativen Klasse“ in deutschen Raumordnungsregionen (Regression). Quelle: BA 2014; SIAB 2014; weitere Sekundärstatistiken; eigene Berechnungen und Darstellung.

\begin{tabular}{|c|c|c|c|c|c|c|}
\hline \multirow[t]{3}{*}{ Standortfaktoren } & \multicolumn{6}{|c|}{ Kreative Klasse } \\
\hline & \multicolumn{2}{|c|}{$\begin{array}{l}\text { Anteil an allen SvB } \\
\text { (Mittel 2003-2006) }\end{array}$} & \multicolumn{2}{|c|}{$\begin{array}{l}\text { Wachstumsrate SvB } \\
\text { (Jahresmittel 2003-2009) }\end{array}$} & \multicolumn{2}{|c|}{$\begin{array}{l}\text { Nettomigrationsrate SvB } \\
\text { (Jahresmittel 2003-2009) }\end{array}$} \\
\hline & (1) & (2) & (3) & (4) & (5) & (6) \\
\hline \multirow[t]{2}{*}{ Arbeitsmarkt } & $0.343^{\star \star \star}$ & $0.349 * \star \star$ & $0.738^{\star \star \star}$ & $0.711^{\star \star \star}$ & $0.883^{\star \star \star}$ & $1.052^{\star \star \star}$ \\
\hline & $(4.239)$ & (4.349) & $(6.904)$ & $(6.024)$ & $(7.296)$ & $(8.689)$ \\
\hline \multirow[t]{2}{*}{ Lebenshaltungskosten } & $0.258^{\star \star \star}$ & $0.279^{\star \star \star}$ & $-0.244^{\star \star}$ & $-0.227^{\star}$ & $-0.328^{\star \star}$ & $-0.286^{\star \star}$ \\
\hline & (3.041) & $(3.569)$ & $(-2.176)$ & $(-1.976)$ & $(-2.583)$ & $(-2.424)$ \\
\hline \multirow[t]{2}{*}{ Toleranz und Offenheit } & $0.266^{\star \star \star}$ & $0.192^{\star}$ & $-0.249 * \star$ & $-0.340^{\star \star}$ & $-0.475^{\star \star \star}$ & $-0.545^{\star \star \star}$ \\
\hline & $(3.309)$ & $(1.873)$ & $(-2.342)$ & $(-2.254)$ & $(-3.951)$ & $(-3.515)$ \\
\hline \multirow[t]{2}{*}{ Kulturelle Vielfalt } & $0.241^{\star \star \star}$ & $0.241^{\star \star \star}$ & $0.328^{\star \star \star}$ & $0.275^{\star \star \star}$ & $0.763^{\star \star \star}$ & $0.818^{\star \star \star}$ \\
\hline & (3.189) & (3.503) & $(3.290)$ & $(2.723)$ & $(6.764)$ & $(7.898)$ \\
\hline \multirow[t]{2}{*}{ Universität } & & 0.045 & & $0.201^{\star \star}$ & & $-0.335^{\star \star \star}$ \\
\hline & & $(0.805)$ & & $(2.463)$ & & $(-4.006)$ \\
\hline \multirow[t]{2}{*}{ Kriminalität } & & -0.042 & & -0.102 & & $0.408^{\star \star \star}$ \\
\hline & & $(-0.521)$ & & $(-0.866)$ & & $(3.394)$ \\
\hline \multirow[t]{2}{*}{ Tourismus } & & $-0.256^{\star \star \star}$ & & 0.021 & & 0.041 \\
\hline & & $(-5.319)$ & & $(0.292)$ & & $(0.569)$ \\
\hline \multirow[t]{2}{*}{ Natur } & & 0.018 & & -0.133 & & $0.193^{*}$ \\
\hline & & $(0.249)$ & & $(-1.243)$ & & (1.758) \\
\hline Korrigiertes $\mathrm{R}^{2}$ & 0.753 & 0.810 & 0.370 & 0.396 & 0.448 & 0.566 \\
\hline F-test & 73.44 & 51.52 & 14.93 & 8.788 & 20.30 & 16.52 \\
\hline Prob $>\mathrm{F}$ & 0.000 & 0.000 & 0.000 & 0.000 & 0.000 & 0.000 \\
\hline
\end{tabular}

Angegeben sind die Beta-Koeffizienten; T-Werte in Klammern; Signifikanzniveau: ${ }^{* \star} p<0.01,{ }^{\star *} p<0.05,{ }^{*} p<0.1$. SvB, Sozialversicherungspflichtig Beschäftigte.

regionalen Standortfaktor zusammengefasst. So umfasst der Faktor „Arbeitsmarkt“ das vorherige Beschäftigungswachstum, das Lohnniveau und die Arbeitslosenquote. Der weiche Faktor „Toleranz \& Offenheit“ setzt sich, in Anlehnung an Floridas „Melting Pot Index“ und „Gay Index“ aus dem Ausländeranteil, dem Anteil von Ehen zwischen In- und Ausländern und dem Anteil von eingetragenen gleichgeschlechtlichen Partnerschaften zusammen. „Kulturelle Vielfalt" speist sich aus der Anzahl der Museumsund Theaterbesucher sowie der absoluten und relativen Anzahl an Bohemiens in einer Region. $\mathrm{Zu}$ den übrigen Standortfaktoren, deren Name selbsterklärend ist, zählen die Lebenshaltungskosten, Universität, Kriminalität, Tourismus und Natur.

Die in Tabelle 3 dargestellten Ergebnisse der Regressionsmodelle unterstützen Floridas Hypothesen nur be- dingt. In sämtlichen Modellen haben die Merkmale der regionalen Arbeitsmärkte einen hochsignifikanten und positiven Effekt, sowohl auf den Anteil als auch auf das Wachstum und die Nettozuwanderung der „Kreativen Klasse“. Dieser harte ökonomische Standortfaktor hat zudem einen größeren Effekt als alle übrigen Faktoren, einschließlich der von Florida besonders betonten weichen Faktoren. Regionen mit hohen Werten für „Toleranz \& Offenheit“"korrespondieren zwar mit hohen Anteilen der Kreativen und bestätigen damit die Ergebnisse von Fritsch/Stützer (2009). Allerdings ist der Effekt auf das Beschäftigtenwachstum der Kreativen und deren Migration entgegen der Erwartungen zum Teil hochsignifikant negativ. Der harte Faktor „Lebenshaltungskosten“ hat einen signifikanten und zum Teil größeren Effekt als der weiche Faktor „Toleranz \& Offenheit“. Dagegen spricht der 


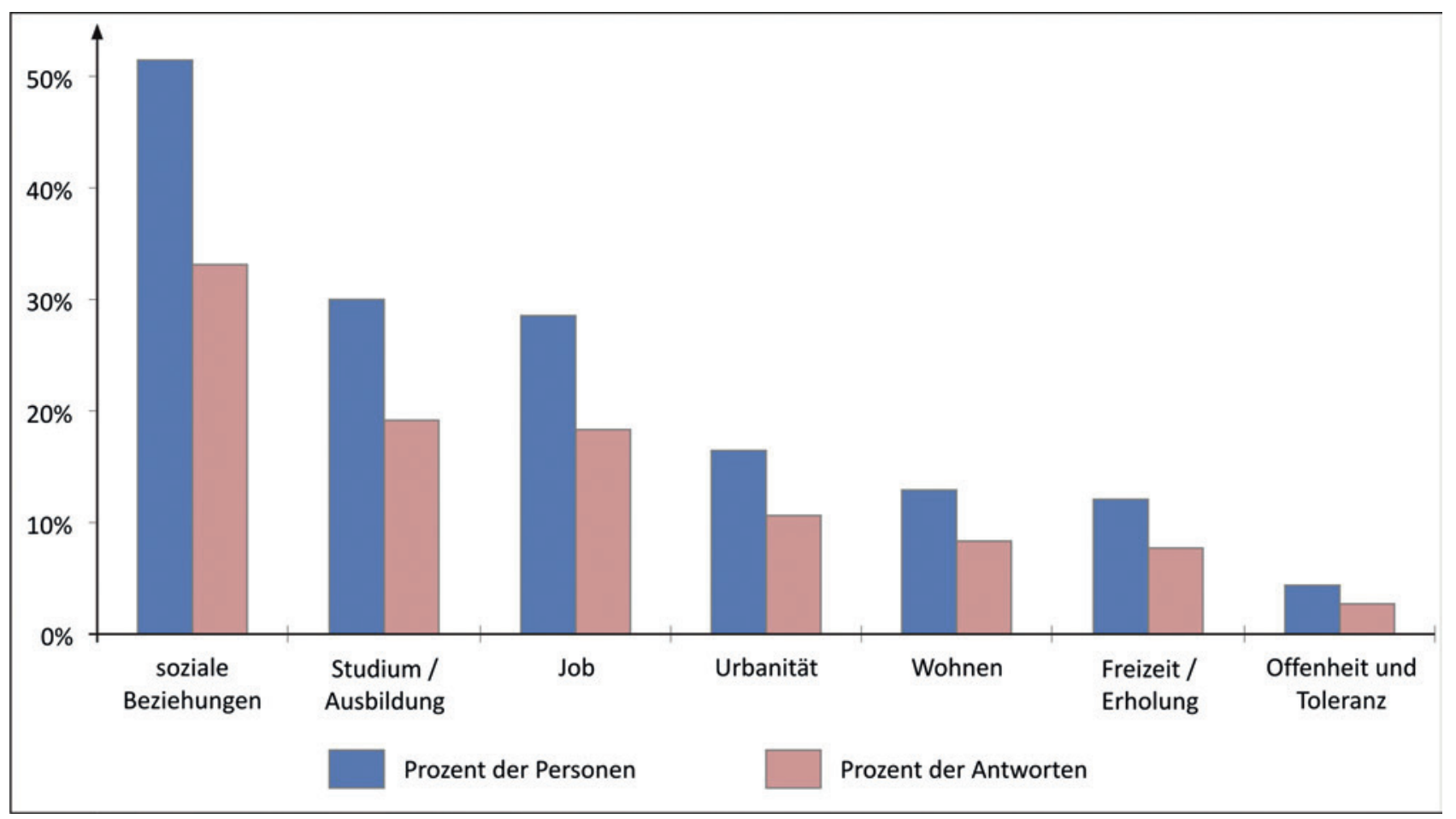

Abbildung 4: Wanderungsmotive Kreativer in drei niedersächsischen Regionen. Quelle: Onlinebefragung 2013 (N=140; Mehrfachantworten waren möglich $=218$ ); eigene Berechnungen und Darstellung.

Einfluss der „Kulturellen Vielfalt“ für Floridas Konzept, denn er ist in allen Modellen hochsignifikant und positiv. Die beobachteten Ergebnisse bleiben robust, wenn Kontrollvariablen oder weitere Faktoren ergänzt werden. Bemerkenswert ist der Einfluss des Faktors „Universität“. Er existiert nicht für den Beschäftigtenanteil der Kreativen, wirkt aber hochsignifikant negativ auf die Nettozuwanderung. Regionen scheinen also durchaus über Wachstum kreativer Beschäftigung von ihren Universitäten zu profitieren, können aber dieses endogene Potenzial nicht zur Gänze ausschöpfen und verlieren Kreative an andere Regionen. Natürlich belegen die gefundenen statistischen Zusammenhänge noch keine Kausalitäten. Selbst mit den zeitlich vorgelagerten Variablen für die erklärenden Standortfaktoren erlaubt eine Analyse auf der aggregierten Makroebene keine Aussagen zu Standortpräferenzen und Migrationsmotive einzelner Individuen der „Kreativen Klasse“. Deshalb werden die sekundärstatistischen Daten um primärstatistische Daten (und damit teils auch um qualitative Methoden) ergänzt, die auf intensiven Befragungen von Mitgliedern der „Kreativen Klasse“ in den Regionen Hannover, Osnabrück und Göttingen basieren (zu den Stichprobenumfängen und Erhebungsmethoden vgl. Kap. 3).

Die 244 kreativen Personen, die an der standardisierten Onlinebefragung teilgenommen haben, wohnen zum
Zeitpunkt der Befragung zu 37,7 \% (Hannover 37,4\%; Göttingen 34,5\%; Osnabrück 40,4\%) in der Stadt, in der sie auch geboren wurden. Somit kommt mehr als die Hälfte der Kreativen aus anderen Städten. Allerdings sind viele der Zuwanderer aus benachbarten Städten der drei Untersuchungsregionen zugezogen. Zwei Fünftel der Zuwanderer nach Osnabrück stammen aus Städten der zugehörigen Raumordnungsregionen, für Hannover (14,7\%) und Göttingen (21,1\%) fällt dieser Anteil deutlich geringer aus. Wird ein $50 \mathrm{~km}$ Radius zugrunde gelegt, erhöht sich der Anteil der der Zuwanderer aus benachbarten Städten noch einmal deutlich (für Hannover auf 34,3\%; Göttingen 50,0\%; Osnabrück 69,2\%). Der Großteil der Befragten wurde in Norddeutschland geboren, nur wenige Kreative stammen aus anderen Teilen Deutschlands oder dem Ausland. Die Hälfte der Befragten war also in der Vergangenheit räumlich mobil, jedoch häufig nur über geringe Distanzen.

Das Migrationsverhalten der Kreativen variiert deutlich mit dem Alter der Befragten. Am häufigsten wandern die Kreativen in ihren frühen und späten Zwanzigern bis frühen Dreißigern, also kurz nach der Schulzeit und zum Ende ihres Studiums oder der Ausbildung. Unter den genannten Wanderungsmotiven spielen üblicherweise $\mathrm{zu}$ den weichen Standortfaktoren gehörende Gründe nur eine sehr geringe Rolle (vgl. Abbildung 4). Im Vordergrund 
standen soziale Beziehungen sowie harte Faktoren wie die Aufnahme einer Ausbildung, eines Studiums oder eine Beschäftigungsmöglichkeit/ein Jobangebot.

Die Tiefeninterviews mit den Kreativen zeigen allerdings, dass deren Standortwahlmotive im Lebenslauf variieren. Insbesondere für Ausbildungsplatzwanderer ist die Wahl der Universität oder des Ausbildungsunternehmens von zentraler Bedeutung, wobei häufig ein Ort in der der Nähe der vorherigen Schulausbildung präferiert wird. Für Jobeinsteiger war die Verfügbarkeit eines Arbeitsplatzes am wichtigsten, wofür die Interviewten i. d. R. bereit waren, die räumliche Nähe zu Familie und Freunden aufzugeben, falls keine Erwerbsalternative vorhanden war bzw. bessere Karriereaussichten in anderen Regionen existierten. Im späteren Lebensverlauf nimmt die Relevanz der Familie deutlich zu. In dieser Phase waren die Interviewten selten mobil, da sie bereits eine Familie gegründet, ein Haus gebaut und eine gute Position in einem Unternehmen inne hatten bzw. selbst eines leiteten. Nur sehr gute Jobangebote konnten noch den Ausschlag für Migration geben.

Die von Florida betonten weichen Standortfaktoren werden nie als primäres Wanderungsmotiv genannt. Zwischenstationen im Lebenslauf der Interviewten waren allerdings häufig dieselben deutschen Metropolen (Berlin, Hamburg, München, Frankfurt oder Köln). Diese Regionen verfügen tatsächlich über eine Vielfalt an weichen Standortfaktoren. Als Wanderungsmotiv wurden diese (auch nach gezielter Nachfrage) allerdings nicht genannt, vielmehr haben diese Städte eine hohe Reputation. Sie sind bekannt als Standort für viele renommierte Unternehmen aus dem Kreativsektor. Diese Zwischenstation hat sich für einige Kreative im späteren Verlauf als vorteilhaft für die eigene Karriere erwiesen. Von diesem letztgenannten Aspekt abgesehen zeigen die Wanderungsmotive der Kreativen in den drei niedersächsischen Regionen keine deutlichen Unterschiede zu den ebenfalls Lebensphasenabhängigen Wanderungsmotiven Nicht-Kreativer bzw. der Binnenwanderer generell (vgl. z. B. Plane et al. 2005, Chen/Rosenthal 2008).

\subsection{Kreative als Unternehmensgründer}

Es ist Konsens in der räumlich ausgerichteten Entrepreneurship-Literatur, dass die Gründung eines Unternehmens primär ein regionales oder gar lokales Ereignis ist, d. h. eher durch regionale denn nationale oder gar supranationale Kontextvariablen beeinflusst wird, neben den selbstverständlichen personenbezogenen Determinanten (für einen Überblick vgl. Sternberg 2009). Dies bedeutet

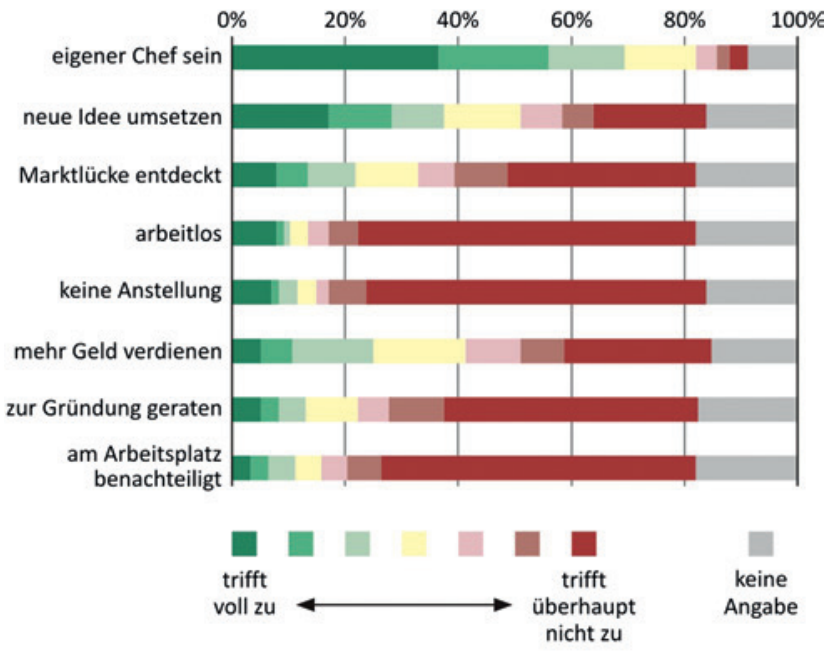

Abbildung 5: Gründungsmotive der Unternehmer der „Kreativen Klasse“ in drei Regionen Niedersachsens. Quelle: Onlinebefragung $2013(\mathrm{~N}=216)$; eigene Berechnungen und Darstellung.

auch, dass Gründer i.d. R. in derselben Region gründen, in der sie zuvor auch abhängig oder gar nicht erwerbstätig waren (vgl. aber differenzierend jüngst Reuschke/ van Ham 2013). Da Kreative aber - so Florida - räumlich besonders mobil und zugleich überdurchschnittlich gründungsaffin sind, ließe sich annehmen, dass es, ggf. auch in Niedersachsen, viele mobile (also regionsexogene) Gründer unter den Kreativen gibt. Dieser Beitrag basiert nicht auf Daten zur regionalen Verteilung kreativer Gründer in Niedersachsen, denn solche Daten liegen flächendeckend, auch andernorts, nicht vor. Vielmehr sollen Erkenntnisse aus den genannten Primärerhebungen unter niedersächsischen Kreativen (vgl. Kap. 3 sowie 4.2) auch für die Frage der Gründungsmotive genutzt werden. In der betreffenden Onlinebefragung wurden auch die Gründungsmotive der Unternehmer erhoben. Sie liefern einen wichtigen Anhaltspunkt über den möglichen Erfolg und die Überlebenschance einer Gründung am Markt. Diesbezüglich hat sich in der Gründungsforschung die Unterscheidung zwischen Opportunity- und NecessityGründern bewährt. Erstere gründen, um eine von ihnen erkannte Marktchance wahrzunehmen, während Letztere aus Mangel an attraktiven Erwerbsalternativen gründen. Opportunity-Gründungen überleben in der Regel länger am Markt, sind wachstumsstärker und gelten als innovativer. Wie Abbildung 5 zeigt, zählen die befragten Gründer der „Kreativen Klasse“ tendenziell zu den OpportunityGründern, auch wenn sich dies aus dem am häufigsten genannten Motiv (,der eigene Chef sein“) nur indirekt ableiten lässt. 
Anders als nach Floridas Konzept zu erwarten wäre, sind auch die zur „Kreativen Klasse“ gehörenden Gründer weit überwiegend endogene, d.h. aus der Region, in der sie aktuell ein Unternehmen führen, stammende Personen. Nur sechs der 58 Interviewten sind unmittelbar vor der Gründung in eine andere Region gewandert. Die genannten Wanderungsmotive hängen zudem kaum mit der Unternehmensgründung selbst zusammen. Vier der sechs exogenen Gründer migrierten wegen privater Motive (folgten ihrem Lebenspartner oder zogen zurück in die Heimatregion in die Nähe von Familie und Freunden). Nur zwei Gründer haben einen Standortwechsel wegen der Gründung getätigt. Dabei waren die Nähe zum (einzigen) Kunden bzw. (bei einer Teamgründung) die vorhandenen Netzwerke eines der drei Gründer in der Region und das damit verbunden Sozial- und Sachkapital ausschlaggebend - nicht aber weiche Standortfaktoren.

Beratungsbedarf besteht laut der Interviewten vor allem in der Anfangsphase bei der Kundenakquise und der Generierung eines ersten und dann konstanten Umsatzes. Neben der finanziellen Belastung waren viele Gründer zunächst mit den organisatorischen und betriebswirtschaftlichen Belangen einer Unternehmung überfordert, denn das betriebswirtschaftliche Wissen aus dem Studium oder der Ausbildung reicht nicht aus. Da kreative Gründer diesbezüglich häufig auf externe Hilfe zurückgreifen müssen, hätten sich viele Gründer retrospektiv einen Mentor oder Coach zu Beginn der Gründung gewünscht. Insbesondere Personen, die selbst Gründungserfahrung in der Branche gesammelt haben, wären eine wichtige Informationsquelle für junge Gründer gewesen - und könnten durch die Wirtschafts- oder Gründungsförderung vermittelt und zumindest partiell finanziert werden. Das speziell auf Gründer aus dem Kreativsektor ausgerichtete Gründungszentrum „Halle 96“ in Hannover erfüllt einige dieser Anforderungen und wurde von vielen Befragten lobend erwähnt, von einer Minderheit allerdings auch bewusst gemieden (negativer Imageeffekt, Nähe zur direkten Konkurrenz).

Zusammenfassend scheinen exogene Gründungen eine vergleichsweise geringe Rolle im Gründungsgeschehen der Kreativbranche in den Untersuchungsregionen zu spielen. Zudem ist das Mobilitätsverhalten der Kreativen mehrheitlich von privaten Motiven gesteuert (vgl. Kap.4.2). Somit ergibt sich insgesamt wenig Spielraum für politische Maßnahmen, um den Zuzug von exogenen Gründern in die eigene Region zu beeinflussen.

Wie die Online-Befragung zeigte, war ein Großteil der Kreativen Gründer und Geschäftsführer ihres Unternehmens, d.h. sie sind auch in der Lage, eine kompetente Bewertung unternehmerischer Standortfaktoren vorzu- nehmen. Neben Unternehmensgründungen lässt sich das endogene Potenzial durch die Unterstützung bestehender Kreativwirtschaftsunternehmen fördern, um deren Wachstum zu stimulieren und damit Arbeitsplätze in der Region zu halten und zu schaffen. Sowohl in der Onlinebefragung als auch in den Interviews haben die Kreativen die Standortfaktoren in den Untersuchungsregionen Hannover, Osnabrück und Göttingen bewertet. Basierend auf der Onlinebefragung illustriert Abbildung 6 die Mittelwerte der Relevanz und der Bewertung der Standortfaktoren für jede der drei im qualitativen Teil untersuchten Regionen. Die gestrichelten Linien markieren die arithmetischen Mittelwerte aller Standortfaktoren der jeweiligen Region. Standortfaktoren mit geringer Relevanz und schlechter Bewertung finden sich im unteren linken Quadranten, jene mit positiver Bewertung, aber geringer Relevanz oben links. Der obere rechte Quadrant zeigt überdurchschnittlich gut und relevant bewertete Standortfaktoren. Durch Maßnahmen der Wirtschaftsförderung adressierbare Standortfaktoren befinden sich im unteren rechten Quadranten, denn sie sind für die Kreativen relevant, werden aber für den betreffenden Standort als relativ „schlecht“ bewertet. Will die Politik etwas verbessern, muss an diesen Faktoren angesetzt werden.

Abbildung 6 zeigt einige wenige Unterschiede der Standortbewertung zwischen den drei Städten (z. B. bei Stadtbild oder -image; Hannover anders als Göttingen und Osnabrück), aber doch auffällig viele Gemeinsamkeiten, auf die sich dieser Absatz konzentriert. Gleichwohl bieten die verfügbaren (hier nur sehr selektiv dargestellten) Daten vielfältige, stadtspezifische Analysemöglichkeiten, auf die hier nicht eingegangen werden kann. Sehr zufrieden sind die meisten Unternehmen mit der Verkehrsanbindung und der Nähe zu Kunden. Dieser Befund der Online-Befragung wird auch in den Interviews bestätigt. Alle Untersuchungsregionen sind gut an den überregionalen Verkehr angebunden und befinden sich in einer zentralen Lage innerhalb Deutschlands. Darüber hinaus sind in allen Regionen hinreichend viele Kunden verortet bzw. der Export von Dienstleistung in andere Region scheint dank moderner IuK-Technologie unproblematisch. Vor allem in Hannover und Osnabrück gibt es mehrere Großunternehmen und Betriebe aus dem Verarbeitenden Gewerbe, die für eine relativ hohe und konstante Nachfrage sorgen.

Bei den weichen Standortfaktoren zeigt sich, dass ein offenes und tolerantes Klima als wichtig und gleichzeitig meist als gegeben erachtet wird. Ähnliches gilt auch für das Image der Region oder des Stadtteils, in dem das Unternehmen ansässig ist. Allein in Hannover scheint das Außen-Image der Region relativ schlecht ausgeprägt zu sein, allerdings haben die Region kennende oder dort 

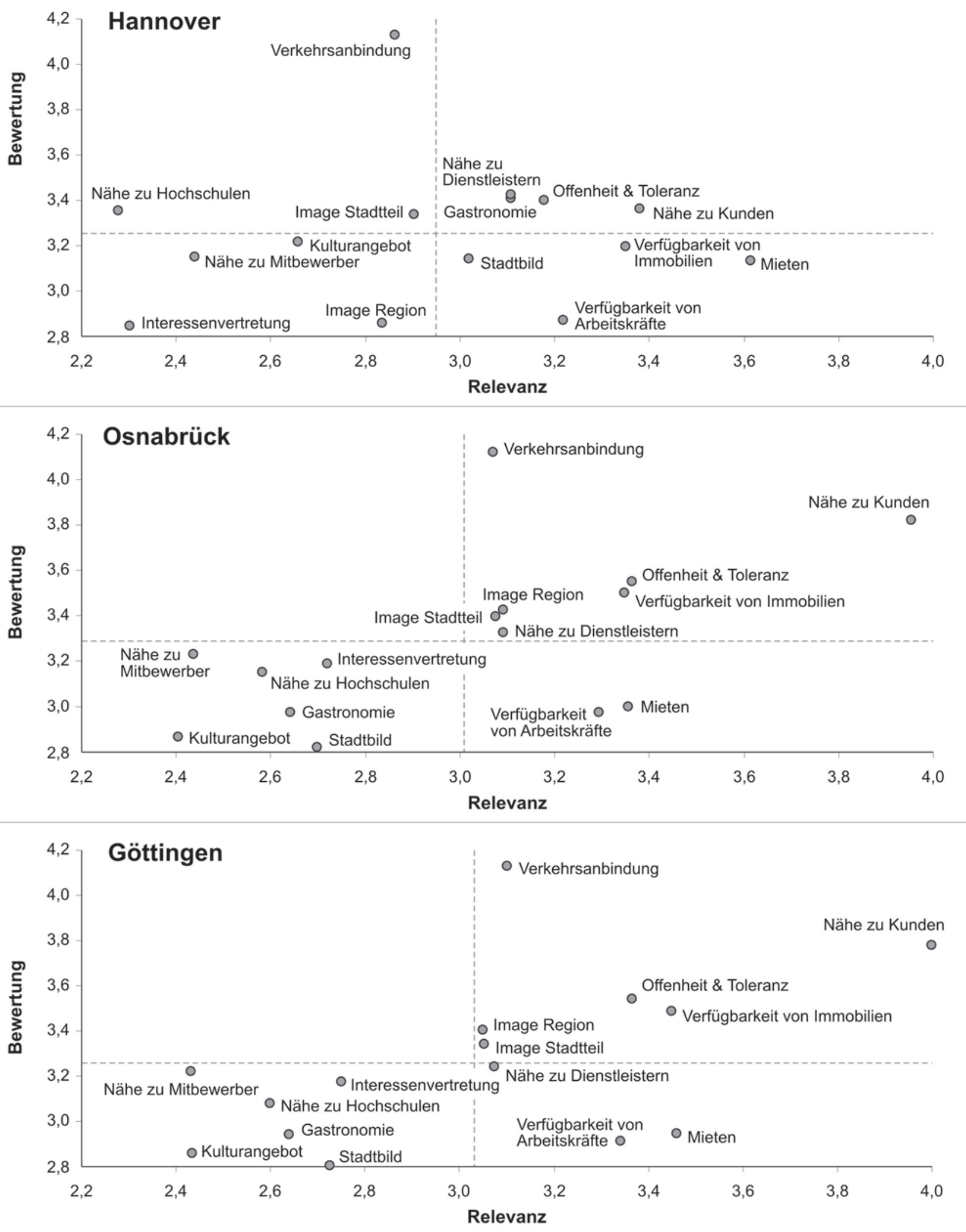

Die Bewertungen basieren auf dem Mittelwert der Einstufungen des jeweiligen Standortfaktors bezüglich seiner Relevanz für sowie seiner Bewertung am Standort durch den Unternehmer auf einer Skala von 1 (unwichtig bzw. sehr schlecht) bis 5 (sehr wichtig bzw. sehr gut).

Abbildung 6: Bewertung und Relevanz der Standortfaktoren in Hannover, Osnabrück und Göttingen. Quelle: Onlinebefragung 2013 ( $N=208$ ); eigene Berechnungen und Darstellung. 
lebende Personen tendenziell ein positives Bild. Die Interviews zeigen klar, dass keine der Untersuchungsregionen eine Reputation als Kreativstandort hat und somit sowohl von Kunden als auch potenziellen Mitarbeiten teils gemieden werden. Dies liegt vor allem daran, dass „Leuchttürme" fehlen, d.h. sehr große und renommierte Kreativunternehmen in der Region, die den Ruf des Standortes prägen könnten. Andere weiche Standortfaktoren, wie das Kultur- und Gastronomieangebot werden in Osnabrück und Göttingen unterdurchschnittlich, aber auch als wenig relevant bewertet.

Alle drei Standorte wurden in den Interviews als „überschaubar“ manchmal „provinziell“ beschrieben. Dies hat einerseits den Vorteil, dass sich die meisten Akteure der Kreativbranche persönlich kennen, was Kooperationen und Kontakte zu Politik und Verwaltung erleichtert. Nachteilig ist, dass dies die Auswahl der möglichen Partner begrenzt. Dies ist in Hannover und Osnabrück, wo sich die Branche in den letzten Jahren stark entwickelt hat, kein Problem, aber in Göttingen scheint derzeit keine „kritische Masse“ vorhanden.

Relevante, aber schlecht bewertete Standortfaktoren sind insbesondere die Verfügbarkeit von qualifizierten Arbeitskräften und der Immobilienmarkt. Laut Interviewten fällt es zunehmend schwer, gutes Personal in der Region $\mathrm{zu}$ halten, da dieses in deutsche Kreativmetropolen abwandere. Die Nähe zu Hochschulen wird in Göttingen und Osnabrück als wenig relevant eingestuft, da dort das Angebot an Studiengängen aus dem Bereich Kreativwirtschaft gering oder nicht vorhanden ist. Selbst in Hannover - mit einem großen Fachbereich für Medien und Design an der Hochschule - wird diese Nähe als wenig relevant erachtet. Offenbar gelingt es trotz vorhandener lokaler Nachfrage nicht, die Absolventen in der Region zu halten. Die Interviews belegen zudem Engpässe bei Ausbildungsberufen, weshalb viele Unternehmen über den eigenen Bedarf hinaus ausbilden.

Die größten Probleme auf dem Immobilienmarkt betreffen die hohen Mieten und die Verfügbarkeit von adäquaten Geschäftsräumen. Viele Kreative wünschen sich mehr Raum z. B. für Ateliers oder Agenturen und erwarten, dass vorhandene Industriebrachen verstärkt für Kreative nutzbar gemacht werden. Trotz vermeintlich zu hoher Mieten sind die meisten Interviewten erstaunlicherweise mit den Lebenshaltungskosten sehr zufrieden.

Insgesamt ist die Einschätzung der Relevanz der Standortfragen über alle drei Städte relativ konstant, die Bewertung der Standorteigenschaften selbst differiert dagegen besonders zwischen Hannover und den beiden kleineren Städten, wobei letztere sich auch bzgl. der Bewertung der Standortfaktoren wenig unterscheiden. Kre- ative zumindest in den drei niedersächsischen Untersuchungsregionen haben also offenbar recht homogene Vorstellungen darüber, welche Standortfaktoren für ihr Unternehmen wichtig und welche weniger wichtig sind. Dabei spielen, abgesehen von „Offenheit und Toleranz“, die weicheren Standortfaktoren (z. B. Stadtbild, Image, Kulturangebot) nur eine untergeordnete Rolle.

\section{Wirtschaftspolitische Implikatio- nen der empirischen Befunde}

Der empirische Teil des Beitrages hat gezeigt, dass in Niedersachsens Regionen einige der Aussagen Floridas bestätigt werden, andere dagegen nicht. Dem Creative ClassKonzept entsprechend, sind Kreative in (den wenigen) urbanen Regionen Niedersachsens absolut und relativ häufiger vertreten als in den ländlichen Regionen. Auch der - verglichen mit anderen westdeutschen Flächenländern - unterdurchschnittliche Besatz an Kreativen in Niedersachsen insgesamt bestätigt Florida, denn Niedersachsen hat einen relativ hohen Beschäftigtenanteil in ländlichen Regionen. Dagegen entspricht der stärkere Zuwachs der kreativen SvB in ländlichen Regionen, verglichen mit nicht metropolitanen Verdichtungsräumen, ebenso wenig den Erwartungen wie die positiv(er)en Nettomigrationsraten mancher ländlicher Regionen. Die Determinanten der Wanderungen der Kreativen, so die Befunde zu den quantitativen und regionsaggregierten Daten, sprechen eher für die Relevanz traditioneller harter, ökonomischer Standortfaktoren als für die von Florida postulierten weichen Standortfaktoren (Toleranz, Offenheit oder Kulturelle Vielfalt), die quantitativ geringere positive oder gar signifikant negative Einflüsse auf Wanderungsraten ausüben. Was die Gründungsaktivität betrifft, lässt sich Floridas Annahme, Gründer machten sich in den Zielregionen ihrer Binnenwanderung selbstständig, nicht bestätigen, denn solche exogenen Gründungen sind unter den kreativen Gründern der drei analysierten Städte sehr selten. Die Gründungsmotive und damit ggf. verbundene Migrationsmotive haben viel mit harten Standortfaktoren sowie individuellen Biographien und Lebensläufen zu tun und gleichen somit eher denen von Nicht-Kreativen. Ein spezifisches Gründungs- und Migrationsverhalten kreativer Gründer, wie von Florida postuliert, ist in Niedersachsen nicht zu beobachten. Die beiden letztgenannten Resultate sind auch Beleg dafür, dass sich Kreative nicht bei jedem Aspekt von den übrigen Beschäftigten (oder Gründungspersonen) unterscheiden. Sie sind also nicht 
bzgl. aller wirtschaftsgeographisch interessanter Themen so spezifisch, wie auch Florida dies oft behauptet.

Was folgt daraus für politische Handlungsempfehlungen an die Adresse jener Wirtschafts-, Gründungs- oder Kulturförderer der Landesregierung bzw. der lokalen Entscheidungsträger, die sich bekanntlich auch in Niedersachsen seit einigen Jahren einer expliziten Unterstützung der Kreativwirtschaft verschrieben haben? Zumindest Florida (2004) hat sich diesbezüglich bekanntlich sehr klar zugunsten einer radikalen Veränderung der Instrumente und Zielgruppen lokaler Wirtschaftsförderung ausgesprochen und insbesondere die lokale Kulturförderung (z.B. Musik) angemahnt. Die nachfolgenden Hinweise basieren i. W. auf den standardisierten oder qualitativen Interviews mit Kreativen aus der Design- und Werbebranche (zu den Erhebungsdetails vgl. Kap.3) in den drei genannten Untersuchungsregionen. Sie sind also nicht unbedingt auf andere Branchen oder Berufe der „Kreativen Klasse“ oder andere Regionen Niedersachsens übertragbar.

Insbesondere zwei hier relevante Handlungsfelder können durch Politikmaßnahmen beeinflusst werden. Einerseits lässt sich das (regions)exogene Potenzial nutzen, also Werbefachleute und Designer aus anderen Regionen attrahieren. Andererseits wäre das (regions)endogene Potenzial besser auszuschöpfen, also Gründungen zu fördern und / oder bestehende Unternehmen der Branche zu unterstützen.

Die Ergebnisse zur Mobilität der Designer und Werbefachleute zeigen die begrenzte Wirksamkeit weicher Standortfaktoren, was die Zuwanderung betrifft. Hauptmotive für interregionale Wanderungen dieser beiden Berufsgruppen sind soziale Beziehungen sowie harte Standortfaktoren wie Ausbildung, Studium und Karrierechancen, jeweils direkt oder indirekt mit dem regionalen Arbeitsmarkt verknüpft. Designer und Werbefachleute verändern ihren Standort insbesondere nach der erfolgreichen Suche nach einem Ausbildungs- oder Studienplatz. Ergo könnten Studien- und Ausbildungsgänge mit Verbindungen zu lokalen Unternehmen dieser beiden Branchen helfen, spätere Designer oder Werbefachleute anzuziehen. Die Interviews legen zudem nahe, dass Qualität der Lehre und Ausstattung der Hochschulen von großer Bedeutung für die Studienortwahl zumindest der befragten Personen waren (und nicht - nur - die Nähe zum Wohnstandort). Eine hohe Reputation der angebotenen Studiengänge kann also helfen, Quantität und Qualität der Studienanfänger und damit die regionale Bindung späterer Designer und Werbefachleute zu erhöhen. Die Verfügbarkeit von bezahlbarem Wohnraum ist dabei allerdings eine notwendige, in manchen Hochschulregionen nicht in ausreichendem Maße gegebene Voraussetzung, wie Abbildung 6 ge- zeigt hat. Hier sollte und kann die Politik handeln, wenn sie die lokale „Kreative Klasse“ nachhaltig unterstützen möchte. Universitäten kommt diesbezüglich in jedem Fall eine wichtige regionale Ankerfunktion zu, denn sie sorgen kontinuierlich für den Nachwuchs der „Kreativen Klasse“ und können potenziell dazu beitragen, dass Absolventen auch nach Verlassen der Universität in der Region verbleiben.

Allerdings, auch dies zeigte die Empirie, sind viele Hochschulregionen u. a. aufgrund mangelnder Absorptionsfähigkeit lokaler Arbeitsmärkte nicht in der Lage, das endogene Potenzial auszuschöpfen und ihre Absolventen dauerhaft an die Region zu binden. Hierzu wären ausreichende und qualitativ hochwertige Karrieremöglichkeiten notwendig. Deshalb sollten Entscheidungsträger eine holistische Strategie unter Einbezug der wichtigsten Stakeholder wie Hochschulen, Kreativunternehmen, Wirtschaftsförderung und Einrichtungen der beruflichen (Aus)Bildung anstreben. Die Kooperation von Hochschulen und Unternehmen kann mittelfristig zu einer besseren Abstimmung von Angebot und Nachfrage auf dem regionalen Arbeitsmarkt führen. Unternehmen können kommunizieren, welche Fähigkeiten und welches Wissen sie von Absolventen erwarten, während Studierende vor Ort frühzeitig Praxiserfahrungen sammeln und ihr eigenes Profil schärfen können. Außerdem wird der Transfer von Wissen aus den Hochschulen in die lokale Wirtschaft gefördert. Aufgaben der Wirtschaftsförderung können die Vernetzung der Stakeholder sowie Vertretung dieser beiden jungen Branchen nach Innen und Außen sein.

Bezogen auf das endogene Potenzial ist die Förderung von Unternehmensgründungen durch Kreative ein sinnvolles und erreichbares Ziel der Politik. Die Interviews mit Designern und Werbefachleuten haben gezeigt, dass schon mit vergleichsweise geringen finanziellen Ressourcen effektive Unterstützung möglich ist. Die Fixkosten sind bei diesen Gründungen zu Beginn i. d. R. niedrig (z. B. sind meist keine eigenen Geschäftsräume notwendig).

Aus den in Kap. 4.3 beschriebenen empirischen Befunden und dem Beratungs- und politischen Handlungsbedarf unter Gründern in der Design- und Werbebranche in den drei untersuchten Regionen Niedersachsens folgt, dass eine Zusammenarbeit zwischen Hochschulen und Wirtschaftsförderung hilfreich sein kann, um insbesondere Gründungsinteressierten das nötige betriebswirtschaftliche Wissen zu vermitteln. Zudem scheint eine finanzielle Unterstützung in der Anfangsphase sinnvoll. Auch die Einbindung von etablierten Gründern aus den beiden genannten Branchen, die ihre spezifischen Erfahrungen weitergeben können, verspricht Erfolg. 
Die befragten Designer und Werbefachleute wünschen sich vor allem mehr Mut der Politik, Projekte in der Region zu wagen. Dies betrifft insbesondere die Schaffung kreativer Freiräume, die Gestaltung des öffentlichen Raums und Imagekampagnen zugunsten der Region und der beiden Branchen. Die Designer und Werbefachleute sehen sich als potenzielle Ideengeber, fühlen sich aber bisher nicht angesprochen bzw. vermuten, dass kreative und gewagte Projekte an bürokratischen Hürden scheitern würden. Die Befragten in Hannover und Osnabrück loben die Initiativen zur Förderung der Kreativwirtschaft mehrheitlich. Es spielt dabei keine Rolle, ob diese Initiativen von Akteuren der Branche selbst oder von der Wirtschaftsförderung initiiert wurden. Wichtig für die Akzeptanz scheint, dass die Designer selbst mitgestalten können. Eine Fortführung und Ausweitung dieser Aktivitäten scheint also wünschenswert.

\section{Ausblick: Wirtschaftsgeographi- sche Aspekte der Kreativwirt- schaft bleiben ein lohnendes Forschungsfeld}

Ziel des Beitrages war es zu prüfen, ob einige zentrale und empirisch bislang außerhalb der USA und insbesondere außerhalb großer Verdichtungsräume nicht belegte Kernaussagen des Creative Class-Konzepts auch für ein Flächenland wie Niedersachsen, das eher von Normalregionen denn von urbanen Metropolen geprägt ist, Gültigkeit besitzen. Der Mehrwert für die Wirtschaftsgeographie liegt aus Sicht der Autoren in der Anwendung eines bislang nur für großstädtische Regionen wissenschaftlich untersuchten Konzepts auch auf nicht-urbane Regionen. Letztere sind nicht nur in Niedersachsen und in Deutschland wesentlich zahlreicher als urbane Metropolen und zudem momentan sehr aktiv, was öffentliche Förderprogramme zugunsten von Kreativen und ihren Unternehmen anbelangt.

Selbstverständlich hat auch der vorliegende Beitrag einige Defizite methodischer und datenbezogener Art. Regionale und flächendeckende Daten zu den Unternehmensgründungen Kreativer wären hilfreich gewesen, um dieses wichtige Potenzial der „Kreativen Klasse“ regional differenzierend bewerten zu können. Die für die Frage der Gründungs- und Wanderungsmotive genutzten qualitativen Daten haben sicher viele Vorteile, ermöglichen aber keine repräsentativen Aussagen für alle Regionen Niedersachsens, nicht einmal für die drei untersuchten Städte.
Die Analyse der Migrationsraten musste sich auf die Untersuchung der deutschen Regionen insgesamt beschränken (keine Daten für Kreise, zu wenige Raumordnungsregionen in Niedersachsen). Zukünftige Untersuchungen sollten versuchen, zumindest für einige Indikatoren kreisbezogene Analysen nur für Niedersachsen durchzuführen.

Unabhängig von der Situation in Niedersachen bieten sich für die räumlich ausgerichtete Forschung zur „Kreativen Klasse“ auch zukünftig zahlreiche lohnende Forschungsthemen. Exemplarisch genannt seien drei Fragen:

- Wie werden die Zuwanderer nach Deutschland die „Kreative Klasse“ angesichts relativ niedriger sektoraler Eintrittsbarrieren beeinflussen?

- Welche (regional unterschiedlichen?) Effekte werden die zumeist noch recht jungen Politikmaßnahmen haben?

- Was passiert mit den zahlreichen Förderinitiativen und der „Kreativen Klasse“, wenn die politische Euphorie um diesen Sektor abebbt (wie bei der Clusterpolitik auch)?

Diese Themen müssen nicht auf die Interpretation der „Kreativen Klasse“ im Sinne Floridas begrenzt bleiben. In vielen Industrieländern außerhalb der USA werden weitere, teils aber auch engere, oft aber andere Definitionen von Kreativen und kreativem Wirtschaften verwendet, die manche der Florida'schen Aussagen gar nicht mehr aufgreifen (oder kennen). Dies mag als Indiz für die Popularität des Grundgedankens Floridas interpretiert werden, aber die Spielarten und Terminologie entfernen sich immer weiter von der Creative Class Floridas. Dies ist besonders augenfällig bei der Adaption der Basisidee der Kreativwirtschaft außerhalb von Industrieländern, wie die spannenden Fragen zur Bedeutung der Kreativwirtschaft für die Rolle der Entwicklungs- und Transformationsländer in der Weltwirtschaft belegen (vgl. dazu den aktuellen UNESCO-Bericht zur "Creative Economy“ weltweit, United Nations 2013 sowie Sternberg, 2017). Kurzum: der Bedarf an theoriegestützter, empirisch fundierter und wirtschaftspolitisch relevanter Forschung zur „Kreativen Klasse“ wird sich verändern, aber er wird vorhanden bleiben.

Es war nicht das Ziel dieses Beitrags, die Konsequenzen der empirischen Befunde für die theoretische Debatte zur „Kreativen Klasse“ zu diskutieren, sondern das Konzept auf eine bislang in empirischen Analysen unterrepräsentierte Normalregion anzuwenden. Dies heißt nicht, dass wir der Ansicht wären, das Creative Class -Konzept sei frei von Mängeln, als Theorie alternativlos und nicht verbesserungswürdig. Vielmehr wirkt das Konzept so wie es ist (mit allen Schwächen - und Stärken) allein dadurch, 
dass es weltweit und auch in Deutschland überaus populär ist, insbesondere bei Vertretern der nationalen und regionalen Wirtschafts- und Kulturpolitik. Aus unserer Sicht ist es eine Aufgabe der empirischen Wirtschaftsgeographie, weit verbreitete theoretische Konzepte auf ihre Anwendung hin zu prüfen sowie etwaige Effekte zu erfassen und zu bewerten. Auch wenn ein Konzept theoretische Schwächen aufweist, entfaltet es bei Verwendung in politischen Strategien Wirkungen, positive wie negative, je nach Zielsystem. Diese Wirkungen können mit den intendierten Zielen z. B. der Wirtschaftspolitik übereinstimmen - oder zumindest partiell von ihnen abweichen. Ein solches Ergebnis empirischer Analysen kann Basis von Handlungsempfehlungen an die Adresse der verantwortlichen Politik(er) sein, z. B. wie die intendierten Ziele eher, effizienter oder effektiver erreicht werden könnten. Ob die empirischen Befunde mit den Thesen des theoretischen Konzepts übereinstimmen, ist für diese Empfehlungen nicht ausschlaggebend, weshalb wir im vorhergehenden Kapitel einige auf unseren qualitativen Daten gestützte Handlungsempfehlungen an die Adresse der Politik formuliert haben.

In späteren Arbeiten kann es sinnvoll sein, auf Basis der hier dargestellten oder anderer neuer empirischer Befunde eine konzeptionelle Weiterentwicklung der „Creative Class“ - Konzepts, vorzunehmen. Insbesondere die Rolle unternehmerischer Selbstständigkeit der „Kreativen Klasse“ könnte detaillierter und aus räumlicher Perspektive beleuchtet werden. Floridas Publikationen beschränken sich diesbezüglich auf eine postulierte (überdurchschnittlich) hohe Gründungsintensität von Kreativen, ohne weitere Begründung. Es wäre spannend zu prüfen, welche Merkmale solche Gründungen und GründerInnen der „Kreativen Klasse“ tatsächlich aufweisen und ob die erwarteten positiven Effekte für die Regionalentwicklung so zwangsläufig sind wie von Florida angenommen. Interessant wäre auch zu überlegen, ob diese Gründungspersonen tatsächlich räumlich so mobil sind wie die Kreativen es laut Florida ganz generell sind oder ob sie, wie GründerInnen in den meisten Fällen, aus guten ökonomischen und privaten Gründen sehr standorttreu sind (vgl. z. B. Dahl/Sorenson 2012). In letzterem Fall könnte die Förderung von kreativen Gründungen zu einem vielversprechenden Instrument einer endogenen Gründungs- und Wirtschaftsförderung in den Regionen werden (vgl. Sternberg 2003), das allerdings die bestehenden Disparitäten zwischen ländlichen und urbanen Regionen eher zementieren denn reduzieren würde.

Angesichts der auch in diesem Beitrag dokumentierten Heterogenität der „Kreativen Klasse“ wäre es sinnvoll, einige der hier behandelten Fragen auch für andere Berufe oder Branchen genauer zu untersuchen, denn zumindest die qualitativen Daten beziehen sich allein auf die Design- und Werbebranche. Diese Heterogenität zeigt sich auch bei der Form selbstständiger Tätigkeit, die bei vielen Bohemiens freiberuflicher Natur, bei den übrigen Mitgliedern der „Kreativen Klasse“ aber zumeist als originäre Unternehmensgründung üblich ist.

Danksagung: Der ausdrückliche Dank der Autoren gilt den Interviewpartnern (Kreative aus den Regionen Hannover, Osnabrück, Göttingen) des qualitativen Teils der Erhebungen. Die Verfasser bedanken sich zudem beim Niedersächsischen Ministerium für Wissenschaft und Kultur für die Unterstützung im Rahmen des Programms „Pro*Niedersachsen“ zwischen 2011 und 2015 (Az 76202-175/10), ohne die diese Publikation nicht möglich gewesen wäre. Den Organisatoren und Teilnehmern des 17. Uddevalla Symposiums 2014 sowie des 3. Research Seminars "Creative Regions in Europe: Challenges and Opportunities” des Regional Studies Association Research Network in Kopenhagen 2012 sei für inspirierende Diskussionsbeiträge zu einem Vortrag über einen Teil der hier präsentierten Befunde gedankt. Schließlich gebührt Jürgen Schneider von der Künstlersozialkasse großer Dank für die Bereitstellung von Regionaldaten.

Funding: Ministerium für Wissenschaft und Kultur Niedersachsen (Grant/Award Number: ,76202-17-5/10`).

\section{Literatur}

Alfken, C. (2015): Ich will nicht nach Berlin! - Life course analysis of inter-regional migration behaviour of people from the field of design and advertising. In: Environment and Planning A, (47), 2187-2203.

Alfken, C. (2016): The spatial concentration and mobility of creative individuals in Germany. Hannover (Dissertation, Naturwissenschaftliche Fakultät der Leibniz Universität Hannover). https:// www.tib.eu/de/suchen/download/?tx_tibsearch_search\%5Bd ocid\%5D=TIBKAT\%3A866805435\&cHash=66321329fa1ec5de1 126f1b8bb45d1ae\#download-mark

Alfken, C./Broekel, T./Sternberg, R. (2015): Factors explaining the spatial agglomeration of the Creative Class - empirical evidence for German artists. In: European Planning Studies, (23)12, 2438-2463.

Andersen, K. V./Bugge, M. M./Hansen, H. K./Isaksen, A./Raunio, M.(2010a): One size fits all ? Applying the creative class thesis onto a nordic context. In: European Planning Studies (18)10, 1591-1609.

Andersen, K. V./Hansen, H. K./Isaksen, A./Raunio, M. (2010b): Nordic city regions in the creative class debate - Putting the 
creative class thesis to a test. In: Industry \& Innovation (17)2, 37-41.

BA (2012): Statistik der Bundesagentur für Arbeit - Sonderauswertung: Arbeitsmarkt in Zahlen, Sozialversicherungspflichtig Beschäftigte nach Berufen (Klassifizierung der Berufe 1988). Durchgeführt im IAB Hannover im Januar 2012.

BA (2014): Statistik der Bundesagentur für Arbeit - Sonderauswertung: Sozialversicherungspflichtig Beschäftigte am Wohnort nach ausgewählten zusammengefassten Berufsgruppen (Klassifikation der Berufe 1988). Durchgeführt im IAB Hannover im März 2014.

Bennett, D. (2010): Creative migration: a Western Australian case study of creative artists. In: Australian Geographer, (41), 117-128.

Book, L./Phillips, D.P. (eds.) (2013): Creativity and entrepreneurship: changing currents in education and public life. Cheltenham, UK, Northampton, MA.

Borén, T./Young, C. (2013): The migration dynamics of the "creative class": evidence from a study of artists in Stockholm, Sweden. In: Annals of the Association of American Geographers, (103)1, 195-210.

Brake, K. (2015): Warum nur arbeiten Kreative so gerne in der Stadt? Über die praktische Relevanz von Stadtaffinität für kreativwirtschaftliche Tätigkeiten. In: DISP, (51), 16-28.

Boschma, R./Fritsch, M. (2009): Creative class and regional growth: empirical evidence from seven European countries. In: Economic Geography, (85)4, 391-423

Bundesministerium für Wirtschaft und Technologie (BMWi) (2009): Gesamtwirtschaftliche Perspektiven der Kultur-und Kreativwirtschaft in Deutschland Kurzfassung. Berlin (= Forschungsbericht 577).

Chantelot, S./Peres, S./Virol, S. (2010): The geography of French creative class: an exploratory spatial data analysis. In: Cahiers du GREThA 2010(16), 1-29.

Chen, Y./Rosenthal, S. S. (2008): Local amenities and life-cycle migration: do people move for jobs or fun? In: Journal of Urban Economics, (64)3, 519-537.

Comunian, R./Faggian, A./Li, Q. C. (2010): Unrewarded careers in the creative class: the strange case of bohemian graduates. In: Papers in Regional Science, (89)2, 389-410.

Dahl, M. S./Sorenson, O. (2012): Home sweet home: entrepreneurs' location choices and the performance of their ventures. In: Management Science, 58(6), 1059-1071. doi:10.1287/ mnsc.1110.1476.

Deutscher Bundestag (2007): Schlussbericht der Enquete-Kommission 'Kultur in Deutschland'. Berlin (=Bundestagsdrucksache 16/7000).

Florida, R. (2002a): Bohemia and economic geography. In: Journal of Economic Geography, (2)1, 55-71.

Florida, R. (2002b): The economic geography of talent. In: Annals of the Association of American geographers, (92)4, 743-755.

Florida, R. (2004): The rise of the creative class. And how it's transforming work, leisure, community and everyday life. New York (Paperback version).

Florida, R. (2005a): Cities and the creative class. London.

Florida, R. (2005b): The flight of the creative class: the new global competition for talent. HarperBusiness, New York, NY.

Florida, R. (2008): Who's your city? How the creative economy is making where to you live the most important decision of your life. New York, NY.
Florida, R./Mellander, C. (2010): There goes the metro: how and why bohemians, artists and gays affect regional housing values. In: Journal of Economic Geography, (10)2, 167-188.

Florida, R./Mellander, C./Stolarick, K. (2008): Inside the black box of regional development - human capital, the creative class and tolerance. In: Journal of Economic Geography, (8)5, 615-649.

Freedman, D./Thornton, A./Camburn, D./Alwin, D./Young-Demarco, L. (1988): The life history calendar: a technique for collecting retrospective data. Sociological Methodology, (18), 37-68.

Fritsch, M./Sorgner, A. (2014): Entrepreneurship and creative professions - a micro-level analysis. In: Sternberg, R./Krauss, G. (eds.): Handbook of Research on Entrepreneurship and Creativity. Cheltenham, 145-176.

Fritsch, M./Stützer, M. (2007): Die Geografie der Kreativen Klasse in Deutschland. In: Raumforschung und Raumordnung, (65)1, 15-29.

Fritsch, M./Stützer, M. (2009): The geography of creative people in Germany. In: Journal of Foresight and Innovation Policy, (5)1, 7-23.

Glaeser, E.L. (2005): Review of Richard Florida's 'The Rise of the Creative Class'. In: Regional Science and Urban Economics (35), 593-596.

Glückler, J., Ries, M., Schmid, H. (2010): Kreative Ökonomie. Perspektiven schöpferischer Arbeit in der Stadt Heidelberg. Heidelberg (= Heidelberger Geographische Arbeiten, Bd.131).

Haisch, T./Klöpper, C. (2007): Creative and academic professions: an analysis of residential selection patterns of highly-skilled persons in the region of Basel, Switzerland. In: Geographica Helvetica (63)2, 75-85.

Hansen, H. K./Niedomysl, T. (2009): Migration of the creative class: evidence from Sweden. In: Journal of Economic Geography, (9)2, 191-206.

Harris, D.A./Parisi, D.M. (2007): Adapting life history calendars for qualitative research on welfare transitions. In: Field Methods, (19), 40-58.

Higgs, P./Cunningham, S. (2008): Beyond the creative industries. Mapping the creative economy. In: Creative Industries Journal, (1), 7-30.

ICG (2011): Kulturwirtschaft in Göttingen. ICG Culturplan Unternehmensberatung $\mathrm{GmbH}$, Göttingen.

Kiese, M. (2008): Mind the gap: Regionale Clusterpolitik im Spannungsfeld von Wissenschaft, Politik und Praxis aus der Perspektive der Neuen Politischen Ökonomie. In: Zeitschrift für Wirtschaftsgeographie (52), 129-145.

Lange, B. (2005): Wachstumsmotor Kreative - eine Kritik an Richard Florida. In: Oswalt, P. (Hrsg.), Schrumpfende Städte Handlungskonzepte. Ostfildern (Ruit), 401-405.

Lange, B./Bürkner, H.-J. (2010): Wertschöpfung in der Kreativwirtschaft: Der Fall der elektronischen Klubmusik. In: Zeitschrift für Wirtschaftsgeographie, (54)1, 46-48.

Lee, S. Y./Florida, R./Acs, Z. (2004): Creativity and entrepreneurship: a regional analysis of new firm formation. In: Regional Studies, (38)8, 879-891.

Markusen, A. (2006): Urban development and the politics of a creative class: evidence from a study of artists. In: Environment and Planning A, (38)10, 1921-1940.

Martin-Brelot, H./Grossetti, M./Eckert, D./Gritsai, O./Kovács, Z. (2010): The spatial mobility of the "creative class": a European 
perspective. In: International Journal of Urban and Regional Research, (34)4, 854-870.

Krätke, S. (2010): “Creative cities" and the rise of the dealer class: a critique of Richard Florida's approach to urban theory. In: International Journal of Urban and Regional Research, (34)4, 835-853.

Marlet, G./van Woerkens, C. (2007): The Dutch creative class and how it fosters urban employment growth. In: Urban Studies, (44)13, 2605-2626.

Möller, J./Tubadji, A. (2009): The creative class, bohemians and local labor market performance. A micro-data panel study for Germany 1975-2004. In: Jahrbücher für Nationalökonomie und Statistik, (229)2-3, 270-291.

McGranahan, D./Wojan, T. (2007): Recasting the creative class to examine growth processes in rural and urban counties. In: Regional Studies, (41), 197-216.

Mossig, I. (2011): Regional employment growth in the cultural and creative industries in Germany 2003-2008. In: European Planning Studies, (19)6, 967-990.

Niedersächsisches Ministerium für Wirtschaft, Technologie und Verkehr (2002): Kulturwirtschaft in Niedersachsen. Hannover.

Niedersächsisches Ministerium für Wirtschaft, Arbeit und Verkehr, Niedersächsisches Ministerium für Wissenschaft und Kultur (2007): Kulturwirtschaftsbericht Niedersachsen 2007. Hannover.

Niedersächsisches Ministerium für Wirtschaft, Arbeit und Verkehr (2015): Monitoring zu ausgewählten wirtschaftlichen Eckdaten der Kultur- und Kreativwirtschaft in Niedersachsen 2014. Hannover.

Peck, J. (2005): Struggling with the creative class. In: International Journal of Urban and Regional Research, (29)4, 740-770.

Plane, D. A./Henrie, C. J./Perry, M. J. (2005): Migration up and down the urban hierarchy and across the life course. In: Proceedings of the National Academy of Sciences, (102)43, 15313-15318.

Pratt, A.C. (2005): Cultural industries and public policy. An oxymoron? In: International Journal of Cultural Policy, (11)1, 31-44.

Reuschke D./Ham, M.van (2013): Testing the 'residential rootedness' hypothesis of selfemployment for Germany and the UK. In: Environment and Planning A, (45), 1219-1239.

Sailer, U./Papenheim, D. (2007): Kreative Unternehmen, Clusterinitiativen und Wirtschaftsentwicklung. Theoretische Diskurse und empirische Befunde aus Offenbach am Main. In: Geographische Zeitschrift, (95)3, 115-137

Scott, A. J. (2010): Jobs or amenities? Destination choices of migrant engineers in the USA. In: Papers in Regional Science, (89)1, 43-63.

SIAB (2014): Stichprobe der Integrierten Arbeitsmarktbiografien (SIAB 1975-2010) am Forschungsdatenzentrum der Bundesagentur für Arbeit im Institut für Arbeitsmarkt- und Berufsforschung. Durchgeführt im IAB Nürnberg im Laufe des Jahres 2014.
Söndermann, M. (2012): Kurzanleitung zur Erstellung einer statistischen Datengrundlage für die Kulturwirtschaft. Statistische Anpassung der Kultur- und Kreativwirtschaft in Deutschland. http://www.kulturwirtschaft.de/wp-content/uploads/2011/01/ Kurzanleitung-KKW_20120124.pdf Creation Date: 2008, abgerufen: 20.04.2013

Söndermann, M. (2014): Monitoring zu ausgewählten wirtschaftlichen Eckdaten der Kultur- und Kreativwirtschaft in Niedersachsen. Köln.

Sternberg, R. (2003): Das Konzept endogener Regionalentwicklung - Implikationen für Existenzgründungen und deren Förderung. In: Sternberg, R. (Hrsg.): Endogene Regionalentwicklung durch Existenzgründungen? Empirische Befunde aus Nordrhein-Westfalen. Hannover, 4-19 (= Arbeitsmaterialien der ARL, 299).

Sternberg, R. (2009): Regional dimensions of entrepreneurship. Boston, Delft (= Foundations and Trends in Entrepreneurship $5(4))$.

Sternberg, R. (2012): Learning from the past? Why creative industries can hardly be created by local/regional government policies. In: Die Erde, (143)4, 293-315.

Sternberg, R. (2017): Creativity support policies as a means of development policy for the global South? A critical appraisal of the UNESCO Creative Economy Report 2013. In: Regional Studies, (51)2, 336-345. DOI: 10.1080/00343404.2016.1174844

Sternberg, R./Krauss, G. (eds.) 2014): Handbook of research on entrepreneurship and creativity. Cheltenham.

Storper, M./Scott, A. J. (2008): Rethinking human capital, creativity and urban growth. In: Journal of Economic Geography, (9)2, 147-167.

Sundbo, J. (2011): Creative artists and entrepreneurship. In: Hindle, K./Klyver, K. (eds.): Handbook of Research on New Venture Creation, Cheltenham, 328-343.

United Nations (2013): Creative economy report 2013. Special edition. Paris

Uricchio, W. (2004): Beyond the great divide, collaborative networks and the challenge to dominant conceptions of the creative industries. In: International Journal of Cultural Studies, (7)1, 79-90.

Vaarst Andersen, K./Lorenzen, M. (2005): The geography of the Danish creative class. Copenhagen Business School.

Wedemeier, J. (2015): Creative professionals, local amenities and externalities: do regional concentrations of creative professionals reinforce themselves over time? In: European Planning Studies, (23)12, 2464-2482.

Wojan, T. R./Lambert, D. M./McGranahan, D. A. (2007): Emoting with their feet: bohemian attraction to creative milieu. In: Journal of Economic Geography, (7)6, 711-736. 